\title{
Médiévales
}

Langues, Textes, Histoire

75 | automne 2018

Traductions du Moyen Âge

\section{Basileus Anglorum. La prétention impériale dans les titulatures royales à la fin de la période anglo- saxonne}

Basileus Anglorum. Imperial claims in regnal styles at the end of the Anglo-

Saxon period

\section{Arnaud Lestremau}

\section{OpenEdition}

\section{Journals}

Édition électronique

URL : https://journals.openedition.org/medievales/9530

DOI : 10.4000/medievales.9530

ISSN : 1777-5892

\section{Éditeur}

Presses universitaires de Vincennes

\section{Édition imprimée}

Date de publication : 15 octobre 2018

Pagination : 197-226

ISBN : 978-2-84292-861-2

ISSN : 0751-2708

\section{Référence électronique}

Arnaud Lestremau, «Basileus Anglorum. La prétention impériale dans les titulatures royales à la fin de la période anglo-saxonne », Médiévales [En ligne], 75 | automne 2018, mis en ligne le 15 octobre 2019, consulté le 23 avril 2022. URL : http://journals.openedition.org/medievales/9530 ; DOI : https:// doi.org/10.4000/medievales.9530 


\section{Arnaud Lestremau}

\section{Basileus Anglorum \\ La prétention impériale dans les titulatures royales à la fin de la période anglo-saxonne}

Bède le Vénérable présente l'Adventus Saxonum comme la migration de trois peuples : Jutes, Angles et Saxons ${ }^{1}$. Après avoir chassé les Bretons, chaque peuple se serait alors divisé et aurait fondé plusieurs royaumes : Kent, Wight, East Anglie, Mercie, Deire, Bernicie, Wessex, Essex, Sussex, etc. En cela, l'histoire des premiers royaumes anglo-saxons s'inscrit dans l'héritage biblique tel qu'il fut transmis par Isidore de Séville, suivant lequel le monde est divisé en gentes ${ }^{2}$. Dans l'idée de Bède, chaque gens dispose d'un territoire, d'un roi et d'un évêque ${ }^{3}$. Aussi, pour légitimer ses prétentions à la tête d'une gens, au $\mathrm{VIII}^{\mathrm{e}}$ siècle, chaque roi fit-il établir une généalogie prestigieuse remontant généralement à Woden ${ }^{4}$. Autour de ces rois, disposant d'un " noyau de tradition ${ }^{5}$ », les royaumes se stabilisèrent, avant de se confronter avec brutalité jusqu'au début $\mathrm{du} \mathrm{IX}^{\mathrm{e}}$ siècle $^{6}$. Les traditions généalogiques

1. BÈDE LE VÉNÉRABLE, Historia ecclesiastica gentis Anglorum, I, xv (Histoire ecclésiastique du peuple anglais, éd. A. Crépin, M. LAPIDGe, P. Monat, P. Robin, Paris, 2005, p. 162 sq.).

2. IsIdORE DE SÉVILlE, Etymologiae, IX, 2, 1 (Étymologies, Livre IX. Les langues et les groupes sociaux, éd. M. ReYdellet, Paris, 1984, p. 40-43). Voir S. ReYnolds, Kingdoms and Communities in Western Europe, 900-1300, Oxford, 1984, p. 258.

3. B. Yorke, «Anglo-Saxon gentes and regna », dans H. W. Goetz, J. Jarnut, W. Pohl éd., Regna and Gentes. The Relationship Between Late Antique and Early Medieval Peoples and Kingdoms in the Transformations of the Roman World, Leyde, 2003, p. 381-408 (p. 390).

4. F. M. Stenton, Anglo-Saxon England, Oxford, 1971, p. 36-37 ; K. SisAm, « AngloSaxon Royal Genealogies », Proceedings of the British Academy, 39 (1953), p. 287-346.

5. R. Wenskus, Stammesbildung und Verfassung. Das Werden der frühmittelalterlichen gentes, Cologne/Graz, 1961. Le caractère en partie littéraire et stéréotypique de ces traditions est désormais bien connu grâce à M. CoumERT, Origines des peuples. Les récits du haut Moyen Âge occidental (550-850), Paris, 2009.

6. S. D. Keynes, « England, 700-900», dans R. McKitTERick éd., The New Cambridge Medieval History, vol. II, c. 700-c. 900, Cambridge, 1995, p. 18-42 ; B. Yorke, Kings and Kingdoms of Early Anglo-Saxon England, Londres, 1990. 
concurrentes et ces guerres incessantes témoignent de la force des sentiments identitaires dans l'Angleterre du haut Moyen Âge ${ }^{7}$.

Bède le Vénérable a aussi fait entrer ces peuples dans l'histoire du salut ${ }^{8}$. Pour ce faire, il a popularisé l'histoire de leur conversion, en insistant sur le rôle auquel leur nom les prédisposait : Angli/Angeli ${ }^{9}$. De la sorte, une identité commune des peuples germaniques insulaires put émerger, avec comme fondements l'usage d'une même langue, une culture similaire, l'inscription dans les mêmes réseaux d'alliances matrimoniales ${ }^{10}$, l'expérience commune de la migration ${ }^{11}$ et de la conversion ${ }^{12}$, ou encore la reprise du cadre romain des provinces méridionales de l'île ${ }^{13}$ et l'opposition subséquente avec les populations celtiques occidentales et septentrionales ${ }^{14}$. $\mathrm{Au} \mathrm{IX}^{\mathrm{e}}$ siècle, le choc des invasions vikings vint renforcer ce sentiment ${ }^{15}$. Dans ce contexte, le terme forgé par Bède prit son essor. Il existait désormais une unité fondée sur une identité ethnique commune (Englacynn, «le peuple anglais ») et sur un territoire commun (Englaland, « la terre des Anglais ») ${ }^{16}$. Boniface, dans une lettre au peuple anglais, vers 738 , souligne cette unité : « Nous sommes d'un seul sang et d'un seul os, issus de la souche de la race des Anglais ${ }^{17}$. » Le patronage pontifical contribua à fixer cette identité de groupe, en mentionnant les Angli et en s'adressant au rex Anglorum ou à la gens Anglica ${ }^{18}$. Acteurs fondamentaux dans la structuration de l'identité

7. P. Wormald, « Engla Lond: the Making of an Allegiance », Journal of Historical Sociology, 7 (1994), p. 1-24 (p. 6).

8. Ibid., p. 13-14 ; N. J. Higham, An English Empire : Bede and the Early Anglo-Saxon Kings, Manchester, 1995.

9. Bède Le VéNérable, Historia ecclesiastica, II, I ; P. Wormald, « Engla Lond... », p. $13 s q$.

10. S. Reynolds, « What Do We Mean by "Anglo-Saxon" and "Anglo-Saxons" ? ", The Journal of British Studies, 24 (1985), p. 395-414 (p. 405).

11. B. Yorke, «Anglo-Saxon gentes...»; N. Howe, Migration and Mythmaking in Anglo-Saxon England, Notre Dame, 2001.

12. P. Wormald, « Engla Lond... », p. 4.

13. ID., «Bede, the Bretwalda and the Origins of the Gens Anglorum», dans P. WormalD, D. A. Bullough, R. Collins éd., Ideal and Reality in Frankish and Anglo-Saxon Society, Oxford, 1983, p. 99-129.

14. D. SCRAGG, «Introduction. The Anglo-Saxons : Fact and Fiction », dans D. SCRAGG et C. WeInBerg éd., Literary Appropriations of the Anglo-Saxons from the Thirteenth to the Twentieth Century, Cambridge, 2000, p. 1-21 (p. 5) ; H. HÄrKE, « Anglo-Saxon Immigration and Ethnogenesis », Medieval Archaeology, 55 (2011), p. 1-28.

15. W. PoHL, "Ethnic Names and Identities in the British Isles : A Comparative Perspective », dans J. Hines éd., The Anglo-Saxons from the Migration Period to the Eighth Century: An Ethnographic Perspective, Woodbridge, 1997, p. 7-32 (p. 23).

16. S. Fоот, "The Making of Angelcynn : English Identity before the Norman Conquest », Transactions of the Royal Historical Society, 6 (1996), p. 25-49 (p. 32).

17. S. Bonifati Epistolae, n ${ }^{\circ} 46$ (éd. M. Tangl, Die Briefe des heiligen Bonifatius und Lullus, Weidmann, 1912).

18. W. D. G. BIRch, Cartularium Saxonicum : a Collection of Charters Relating to Anglo-Saxon History, Londres, 1885-1893, $\mathrm{n}^{\circ} 119, \mathrm{n}^{\circ} 614, \mathrm{n}^{\circ} 615, \mathrm{n}^{\circ} 915, \mathrm{n}^{\circ} 916, \mathrm{n}^{\circ} 1070$ et 
anglo-saxonne, les souverains insistèrent sur ce terme. Ce faisant, ils asseyaient leurs prétentions territoriales sur une adéquation totale entre gens et regnum, l'appartenance ethnique commandant l'allégeance politique ${ }^{19}$. Aussi retrouve-t-on cet usage éphémère dans les chartes d'Offa de Mercie (757-796), qui prétendait dominer tous les Anglo-Saxons ${ }^{20}$. Pour autant, jamais aucun royaume ne put obtenir mieux qu'une suprématie passagère sur ses voisins avant le dernier quart $\mathrm{du}_{\mathrm{IX}}^{\mathrm{e}}$ siècle, au moment où débute un vaste mouvement, intellectuel et logistique, d'unification du royaume des Anglais qui se poursuit sans interruption sérieuse jusqu'à la conquête normande. Mais peut-on se contenter de parler de « royaume»?

Pour désigner la suprématie, même passagère, d'un roi anglo-saxon sur ses voisins, Bède utilisait le mot imperium $^{21}$. Le mot « empire » pose toutefois problème tant les «formes », « configurations » et « expériences » impériales constituent un « répertoire » ouvert. Il est donc impossible de le réduire à un concept simple ${ }^{22}$. Le pouvoir impérial repose sur la sacralité du souverain ${ }^{23}$, mais " l'espace apparaît comme un élément de définition plus essentiel ${ }^{24} \gg$. Ce critère implique la domination de territoires $\operatorname{vastes}^{25}$ ou à défaut « le souvenir d'un pouvoir étendu dans l'espace ${ }^{26}$ ».

$\mathrm{n}^{\circ} 1271$; D. Whiтelock, Councils and Synods with other Documents Relating to the English Church, 1 : AD 871-1204, Oxford, 1981, $\mathrm{n}^{\circ} 70, \mathrm{n}^{\circ} 74$ et $\mathrm{n}^{\circ} 75$; N. BROоKs, « Canterbury, Rome and English Identity », dans D. Bullough et J. Smith éd., Early Medieval Rome and the Christian West, Leyde, 2000, p. 221-247.

19. S. Reynolds, Kingdoms and Communities..., p. 261.

20. P. H. SAwYer, Anglo-Saxon Charters, Londres, 1968 : S 54, S 104, S 108, S 109, S 110, S 111, S 121, S 132, S 133, S 145, S 146 et S 1178 ; E. John, Orbis Britanniae, and Other Studies, Leicester, 1966, p. 24-26.

21. Bède le Vénérable, Historia ecclesiastica, L. II, cap. V. Voir J. L. Nelson, « Kingship and Empire in the Carolingian World», dans R. McKitTerick éd., Carolingian Culture : Emulation and Innovation, Cambridge, 1994, p. 52-87. La Chronique anglo-saxonne donne pour sa part le titre de bretwalda aux rois qui obtinrent une telle suprématie: The Anglo-Saxon Chronicle MS A, sub anno 827 (éd. J. M. BATELY, The Anglo-Saxon Chronicle. A Collaborative Edition, vol. III, MS A, Oxford, 1986). Voir P. WormaLD, « Bede, the Bretwalda..., p. 99-129.

22. T. MÉNISSIER éd., L'Idée d'empire dans la pensée politique, historique, juridique et philosophique, Paris, 2006, p. 11 ; F. Hurlet et J. Tolan, « Conclusions. Vertus et limites du comparatisme », dans F. Hurlet éd., Les Empires : Antiquité et Moyen Âge. Analyse comparée, Rennes, 2008, p. 239-250 (p. 239) ; F. MADELINE, "L'empire et son espace. Héritages, organisations et pratiques », Hypothèses, 11 (2008), p. 213-225 (p. 215-216) ; J. Burbank et F. CoOPer, Empires. De la Chine ancienne à nos jours, Paris, 2011, p. 16.

23. G. DaGRon, «Empires royaux, royautés impériales : lectures croisées sur Byzance et la France médiévale », dans Summa : Dieter Simon zum 70. Geburtstag, Francfort, 2005, p. 81-97 (p. 82 et p. 88-89) ; F. Hurlet et J. Tolan, « Conclusions... », p. 239 ; F. Madeline, «L'empire et son espace... », p. 217 ; J. BuRBANK et F. CoOPER, Empires..., p. 33 ; M. GRENET et A. Rappas, « Empire », dans D. Albera, M. Crivello, M. Tozy éd., Dictionnaire de la Méditerranée, Arles, 2016, p. $452-463$ (p. 460).

24. M. Duverger éd., Le Concept d'empire, Paris, 1980, p. 8.

25. T. Ménissier éd., L'Idée d'empire..., p. 15 ; J. Burbank et F. CoOper, Empires..., p. 23 ; F. MAdeline, «L'empire et son espace... », p. 223.

26. J. Burbank et F. CoOper, Empires..., p. 23. 
S'il convient de ne pas réduire l'empire à son caractère impérialiste et expansionniste, Thierry Ménissier note que « la plupart des empires [...] se sont effectivement fondés [...] sur la tentative de dominer d'autres peuples $^{27} \gg$. Cela se traduit donc souvent par la propension de l'empire à dominer « divers autres royaumes et divers autres rois », pour reprendre la définition d'Isidore de Séville ${ }^{28}$, et, in fine, par ses prétentions universelles ${ }^{29}$. Dans tous les cas étudiés, les empires apparaissent également comme des structures souples et hybrides, maintenant une tension entre intégration et différentiation de ses composantes internes, mettant en jeu des formes de domination formelle et informelle et disposant en conséquence de marges/ périphéries mal intégrées plutôt que de frontières clairement définies ${ }^{30}$. En nous appuyant sur ces travaux, notre propos est donc de montrer comment la royauté «west-saxonne » fut, comme d'autres « états médiévaux ${ }^{31}$ », une construction hybride utilisant des traits, des discours et un imaginaire impériaux, rassemblés au gré d' " opportunités contingentes ${ }^{32}$ ». Quels discours impériaux sont construits à la cour de Winchester?

Pour répondre à cette question, il convient de partir de «l'ordre des faits » qu'imposent les discours construits dans l'entourage des rois ${ }^{33}$. Il s'agit donc de compléter la proposition établie par George Molyneaux en 2011 lorsqu'il se détournait des détails pour se concentrer sur la relation générale des rois anglais avec leurs voisins ${ }^{34}$. Nous proposons ici la démarche inverse, en partant de la documentation. Entre 871 et 1066, quelques 700 chartes royales (dont 400 sont authentiques), un peu plus de 22000 monnaies (dont

27. T. MÉNISSIER éd., L'Idée d'empire ...,p. 14 (voir aussi p. 12) ; J. Burbank et F. COOPER, Empires..., p. 23.

28. ISIDORE DE SÉVILLE, Etymologiae, IX, 3,3: « regna cetera ceterique reges velut adpendices istorum habentur ». Voir T. MénIssier éd., L'Idée d'empire..., p. 13 ; F. HurLet et J. Tolan, «Conclusions... », p. 239 ; J. Burbank et F. Cooper, Empires..., p. 14 ; M. Grenet et A. Rappas, «Empire »...,p. 461.

29. P. Veyne, "L'Empire romain », dans M. Duverger éd., Le Concept d'empire, Paris, 1980, p. 122 ; G. Dagron, « Empires royaux... », p. 86-87 ; F. Hurlet et J. Tolan, «Conclusions... », p. 239 ; F. Madeline, « L'empire et son espace... », p. 217-218.

30. G.DAGRon, «Empires royaux...»,p. 83-85; F. Hurlet et J.TOlan, «Conclusions...», p. 239 ; F. MADELINE, «L'empire et son espace... », p. 217-218 ; G. MarTinez-Gros, Brève histoire des empires. Comment ils surgissent, comment ils s'effondrent, Paris, 2014 ; J. Burbank et F. COOPER, Empires..., p. 28-30, 32, 34.

31. G. DAGRon, « Empires royaux... », p. 81.

32. L. Gerbier, Les Raisons de l'empire, Paris, 2016, p. 16, en référence à J. Perez, « La idea imperial de Carlos V », dans F. Sanchez-Montes Gonzalez et J. L. Castellano Castellano, Carlos V, Madrid 2001, p. 239-250.

33. L. Gerbier, Les Raisons de l'empire ..., Paris, 2016, p. 21-25.

34. G. Molyneaux, «Why Were Some Tenth-Century English Kings Presented as Rulers of Britain ?», Transactions of the Royal Historical Society, 21 (2011), p. 59-91 (p. 65 pour l'exposé de cette approche). 
l'avers de 2800 est à peu près lisible) et 32 codes de lois sont préservés ${ }^{35}$. Dans ces documents, les titulatures royales n'adoptent pas une formulation uniforme. Elles incorporent parfois une liste de peuples, parfois une unité géographique plus ou moins définie. Certains en ont conclu que ces deux types de titulatures étaient totalement interchangeables ${ }^{36}$. En effet, à ce moment, l'application d'un droit n'est liée ni à l'ethnicité de l'acteur, ni à une attache territoriale, mais au statut de sujet du roi ${ }^{37}$. Nous considérons, pour notre part, que cette ambiguïté a pu aider à négocier l'intégration de peuples marginaux dans la nouvelle construction politique du royaume des Anglais, en laissant planer un doute sur la nature du pouvoir revendiqué par les rois de Winchester. Nous nous intéresserons d'abord à l'utilisation des titulatures dans la dynamique d'unification du royaume des Anglais, avant de poser la question de leur caractère impérial.

\section{L'unification des peuples anglo-saxons dans un royaume unique}

Le dernier roi affublé du titre de bretwalda fut Ecgberht, roi des WestSaxons (802-839), à l'occasion de la conquête de la Mercie, en 829. Au cours de la même année, il aurait reçu la soumission des Northumbriens, avant d'entreprendre, en 830 , une expédition en Galles ${ }^{38}$. Mais dès 830 le pouvoir d'Ecgberht recule : le Kent est confié à son fils, Æthelwulf, et Wiglaf redevient le roi indépendant de la Mercie ${ }^{39}$. Lorsqu' Æthelwulf accéda au pouvoir, en 839, « il donna à son fils, Æthelstan, le royaume de Kent, d'Essex, de Surrey et de Sussex ${ }^{40} »$. Lorsqu' Ethelwulf mourut, en 855 , Æthelstan avait déjà disparu, de sorte que ce furent « Ethelbald [qui

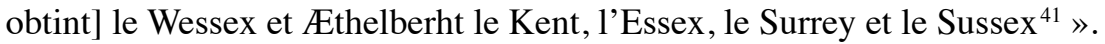
Ainsi, le roi qui gouvernait le Wessex n'était jamais réellement à la tête des royaumes subalternes du sud-est de l'Angleterre ${ }^{42}$.

35. Pour les chartes, nous renvoyons à P. H. SAwYer, Anglo-Saxon Charters... [cidessous abrégé $\mathrm{S}$ suivi du numéro de la charte]. Les lois sont éditées par F. Liebermann, Die Gesetze der Angelsachsen, Halle, 1898. Les monnaies anglo-saxonnes sont consultables sur le site : Corpus of Early Medieval Coin Finds et Sylloge of Coins of the British Isles, 2011 [en ligne] https://emc.fitzmuseum.cam.ac.uk/index.php [consulté le 2 octobre 2018].

36. F. BARLow, Edward the Confessor, New Haven/Londres, 1997, p. 136.

37. J. Campbell, The Anglo-Saxon State, Londres, 2000, p. 40-41 ; S. D. Keynes, «Edgar, rex admirabilis » dans D. G. SCRAGG éd., Edgar, King of the English, 959-975 : new interpretations, Woodbridge, 2008, p. 3-59 (p. 25).

38. The Anglo-Saxon Chronicle MS A, sub annis 827 et 828. Barbara Yorke (Kings and Kingdoms..., p. 96) nuance le sens à donner à cette « soumission ».

39. F. M. Stenton, Anglo-Saxon England..., p. 233-235.

40. The Anglo-Saxon Chronicle MS A, sub anno 836.

41. Ibid., sub anno 860.

42. Voir B. Yorke, «The Bishops of Winchester, the Kings of Wessex and the Development of Winchester in the Ninth and Early Tenth Centuries », Hampshire Field Club and Archaeological Society, 40 (1984), p. 61-70 (p. 67-68). 


\section{La première unification sous Alfred}

Ce paysage change en 860 . Avec les règnes d'Æthelberht (860-865) et Æthelred ${ }^{\text {er }}$ (865-871), le roi de Wessex domine un large territoire qui inclut la totalité des royaumes situés au sud de la Tamise. Toutefois, la situation est confuse : les Vikings tentent d'attaquer Winchester en $860^{43}$, pillent le Kent en $865^{44}$ et la Grande Armée arrive en East Anglia l'année suivante, menaçant sérieusement le royaume d' Ethelred $^{45}$. De fait, le roi passe le début de l'année 871 sur les champs de bataille, essuie de cuisants revers à Reading, Basing et Meretun, avant de s'éteindre au printemps, «peu après Pâques ${ }^{46} »$. À leur prise de pouvoir, la situation politique et militaire n'est donc guère favorable à ces rois. En conséquence, ils se dispensent des innovations que d'autres ont tentées avant eux. Ils conservent le simple titre de rex Occidentalium Saxonum ${ }^{47}$ et, suivant la situation, y adjoignent leur titre de rois du Kent ${ }^{48}$. De la même manière, Alfred (871-899) arrive au pouvoir dans une situation compliquée. Dans ses chartes, sur ses monnaies et dans d'autres documents, il reprend la titulature qui avait cours avant lui : il est rex Saxonum, rex Westsaxonum ou rex Occidentalium Saxonum ${ }^{49}$. Quelques successeurs d'Alfred utilisent encore parfois ce style dans la suscription de leurs chartes ${ }^{50}$ et sur leurs monnaies ${ }^{51}$.

Cependant, à mesure que le Wessex desserre l'étau scandinave et qu'il incorpore les royaumes subalternes du sud-est de l'île, à compter du dernier quart du $\mathrm{IX}^{\mathrm{e}}$ siècle, les titulatures des souverains évoluent pour rendre compte de l'unification des peuples germaniques de Grande-Bretagne derrière un même pouvoir ${ }^{52}$. Après la victoire d'Edington (878), le traité de Wedmore $(878)^{53}$ et la prise de Londres $(886)^{54}$, Alfred retrouve la pleine

43. The Anglo-Saxon Chronicle MS A, sub anno 860.

44. Ibid., sub anno 865 .

45. Ibid., sub anno 867 .

46. Ibid., sub anno 871. F. M. Stenton, Anglo-Saxon England..., p. 248-249.

47. Rex Occidentalium Saxonum ou rex Saxonum : Æthelberht (S 329, S 333), Æthelred (S 334, S 335, S 336, S 338, S 339a, S 340, S 341, S 342).

48. Rex Occidentalium Saxonum necnon [ou necnon et ou seu] Cantauuariorum : Æthelberht (S 266, S 327, S 330, S 331, S 332), Æthelred (S 338, S 339).

49. S 343-S 346 et S 348-S 350. Voir aussi Code d'Alfred, § 49.10, les monnaies portant comme titre rex Saxonum ou rex Sax (nous en avons trouvé 7, de types N 629, N 632 et N 647, pour moitié antérieures à 880 à coup sûr) et The Anglo-Saxon Chronicle MS A, sub anno 885). Voir S. D. Keynes et M. LAPIDGE, Alfred the Great, Londres, 2004, p. 227-228.

50. S 372, S 516, S 636 et S 654.

51. Æthelstan sur 9 monnaies de types N 671 et N 672 .

52. S. D. Keynes, «Edward, King of the Anglo-Saxons », dans N. J. Higham et D. H. Hill éd., Edward the Elder, 899-924, Londres, 2001, p. 40-66 (p. 57-62).

53. The Anglo-Saxon Chronicle MS A, sub anno 878 ; F. M. Stenton, Anglo-Saxon England..., p. 255-257.

54. Ibid., sub anno 886 ; F. M. Stenton, Anglo-Saxon England..., p. 258-259. Pour les nuances qui s'imposent sur ces faits, voir S. D. KeYNES, « Alfred the Great and the Kingdom 
maîtrise du royaume qu'avait tenu Ecgberht : Wessex, Kent, Sussex, Surrey, Essex et une partie de la Mercie. Un tel héritage est fermement revendiqué dans les lois édictées par Alfred. Dans le prologue de ces lois, il se met en scène marchant dans les pas des grands rois du sud de l'île :

Moi, le roi Alfred, ai regroupé ceci et ai demandé que ceci soit écrit, à savoir les textes nombreux que nos prédécesseurs respectaient, tout au moins ceux qui me plaisaient; et beaucoup de ceux qui ne me plaisaient pas, je les ai écartés avec le conseil de mon witan [...]. J'ai donc rassemblé ces textes qui remontent ou bien au temps du roi Ine, mon parent, ou bien au temps du roi Offa de Mercie, ou bien au temps d'Æthelberht, qui en premier fut baptisé parmi les Anglais ${ }^{55}$.

Alfred se place donc dans l'héritage direct du premier roi chrétien du Kent, Æthelberht (vers 560-616), héros de Bède le Vénérable ${ }^{56}$, dont le royaume est passé sous la coupe du Wessex depuis la victoire d'Ecgberht contre les Merciens en 825, lors de la bataille d'Ellandun ${ }^{57}$. Il reprend bien sûr à son compte les apports d'Ine, roi et législateur du Wessex (688726), dont on considère généralement qu'il était le frère d'Ingild (672718), l'ancêtre d'Alfred au sixième degré ${ }^{58}$. Plus bizarrement, il reprend également à son compte la gloire d'Offa, grand roi de Mercie (757-796), rival traditionnel du Wessex, mais surtout allié de Charlemagne ${ }^{59}$. Tous ces éléments étant mis bout à bout, la Chronique anglo-saxonne conclut qu'Alfred domine alors « la totalité du peuple anglais ${ }^{60} »$.

Cette hégémonie permet à Alfred de revendiquer la domination de plusieurs peuples, mais au sein d'un même royaume, ou d'un même peuple, réparti dans plusieurs royaumes. Il choisit alors de mettre en avant l'unité du peuple anglo-saxon pour garantir l'unité de son royaume. Cette conception politique s'impose donc dans le matériau diplomatique et narratif : Alfred est désormais rex Angulsaxonum ${ }^{61}$. Six chartes, sans doute authentiques,

of the Anglo-Saxons », dans N. Guenther-Discenza et P. E. Szamarch éd., A Companion to Alfred the Great, Leyde, 2014, p. 13-46 (p. 22-24).

55. Code d'Alfred, $\$ 49.9$.

56. Bède Le Vénérable, Historia ecclesiastica, I, XXV à II, v ; B. Yorke, Kings and Kingdoms..., p. 28-29.

57. The Anglo-Saxon Chronicle MS A, sub anno 823 ; F. M. Stenton, Anglo-Saxon England..., p. 231.

58. Bède Le Vénérable, Historia ecclesiastica, V, viI ; B. Yorke, Wessex in the Early Middle-Ages, Leicester/Londres, 1995, p. 59 sq.

59. F. M.Stenton, Anglo-Saxon England...,p. 206-224 ; B. Yorke, Kings and Kingdoms..., p. 112-117.

60. The Anglo-Saxon Chronicle MS A, sub anno 886. Voir F. M. Stenton, Anglo-Saxon England..., p. 232-233 ; E. John, Orbis Britanniae..., p. 44-46.

61. F. M.Stenton, Anglo-Saxon England...,p. 232-233 ; S. D. Keynes et M. LaPidge, Alfred the Great..., p. 228 ; S. D. KeYNES, « King Alfred and the Mercians », dans M. A. S. BLACKBuRn, 
conservées dans les archives de six monastères, suggèrent cette évolution ${ }^{62}$. De même, le biographe du roi, Asser, dont la Vita Alfredi remonte sans doute à l'an 893 , multiplie les usages de ce titre ${ }^{63}$. Ce dernier conforte l'alliance entre West-Saxons et Merciens au sein d'un même peuple contre la menace danoise ${ }^{64}$. Les successeurs d'Alfred n'hésitent donc pas à l'utiliser à leur tour ${ }^{65}$.

Toutefois, la réapparition du terme Angli dans les représentations politiques date aussi du règne d'Alfred le Grand (871-899) ${ }^{66}$. L'hégémonie d'Alfred lui permet en effet de se placer dans l'héritage de lointains et prestigieux prédécesseurs, comme Offa ${ }^{67}$ et Æthelwulf ${ }^{68}$. Il apparaît donc à l'occasion comme rex Anglorum, en particulier dans une lettre de Foulques de Reims dans les années 880 et dans quelques sources insulaires ${ }^{69}$. La production de généalogies spécifiques, remontant jusqu'à l'époque biblique, constituèrent le soubassement d'une telle revendication ${ }^{70}$, puisqu'elles permettaient de transformer Alfred en chef d'une famille légitimée par cet ancrage dans la tradition biblique. Dès lors, la mise en ordre de l'histoire dynastique était en adéquation avec l'histoire de la gens elle-même, de sorte

D. N. Dumville éd., Kings, Currency, and Alliances : History and Coinage of Southern England in the Ninth Century, Woodbridge, 1998, p. 1-45.

62. S 346-348 et S 354-356. Voir S. D. Keynes et M. LaPIDGE, Alfred the Great..., p. 228 ;

D. Whitelock, «Some Charters in the Name of King Alfred », dans Saints, Scholars, and Heroes. Studies in Medieval Culture in Honor of Charles W. Jones, Collegeville, 1979, vol. I, p. 77-98.

63. Asser, Vita Alfredi, 1, 13, 21, 64, 67, 71, 73, 83 et 87 (Histoire du roi Alfred, éd. et trad. A. GAutier, Paris, 2013).

64. S. D. Keynes, « King Alfred and the Mercians... », p. 26.

65. S 346-S 348, S 354-S 356, S 358-359, S 362-366, S 368-369, S 373, S 375-378, S 380-384, S 392, S 394, S 396-397, S 427, S 472-473, S 479, S 520, S 549, S 566, S 569, S 572, S 590, S 598, S 606, S 608, S 624, S 633, S 635, S 644, S 651, S 672, S 830, S 890, S 898, S 931. Pour cette reprise, voir par exemple S. Foot, Ethelstan : the First King of England, New Haven, 2011, p. 25-26.

66. S. Foot, «The Making of Angelcynn... »; R. DAvies, The First English Empire. Power and Identities in the British Isles, 1093-1343, Oxford, 2000, p. 49-51.

67. Les chartes S 54, S 104, S 108, S 109, S 110, S 111, S 121, S 132, S 133, S 145, S 146 et S 1178 d'Offa utilisent ce titre. Néanmoins, ces chartes sont, sauf exception, considérées comme très douteuses. Seule la charte S 109 de 775-777 est sans doute authentique.

68. Dans le Liber Vitae de Brescia et dans une lettre du pape Léon IV, Æthelwulf figure sous le titre de rex Anglorum. Voir Liber Vitae de Brescia, f. 27 (D. Geuenich, U. Ludwig, A. Angenendt éd., Der Memorial- und Liturgiecodex von San Salvatore, Hanovre, 2000) ; et Monumenta Germaniae Historica. Epistolae Karolini Aeui, t. III, Berlin, 1899, p. 602. C'est aussi le cas dans deux chartes très suspectes : S 318 et S 320 .

69. Lettre de Foulques à Alfred dans D. WhiтеLоcк, English Historical Documents, c. 500-1042, New York, 1955, $\mathrm{n}^{\circ} 225$; The Anglo-Saxon Chronicle MS A, sub anno 900 ; monnaies de type Two Emperors ; F. M. STENTON, Anglo-Saxon England..., p. 211-212.

70. K. SisAm, «Anglo-Saxon... », p. 299-301; C. Davis, « Cultural Assimilation in the Anglo-Saxon Royal Genealogies », Anglo-Saxon England, 21 (1992), p. 23-36 (p. 28-31) ; D. N. Dumville, «The West Saxon Genealogical Regnal List and the Chronology of Early Wessex », Peritia, 4 (1985), p. 21-66 (p. 61-66). 
qu'Alfred et ses successeurs pouvaient revendiquer une autorité de droit sur l'ensemble des Anglo-Saxons ${ }^{71}$.

On explique l'usage de la notion de gens Anglorum par la multiplication des références à Bède à l'époque d'Alfred. La transmission du texte de Bède par la voie de manuscrits ${ }^{72}$, par une traduction vernaculaire peut-être commandée par Alfred lui-même ${ }^{73}$ et par le biais de la Chronique anglosaxonne, probablement produite elle aussi dans le contexte de la rénovation alfrédienne $^{74}$, contribuèrent grandement à populariser ce terme. Or, dans le contexte des guerres vikings, l'unité de la gens Anglorum agissait comme un discours de légitimation des prétentions west-saxonnes ${ }^{75}$. En somme, les chrétiens de langue germanique étaient appelés à reprendre pour eux le nom d'Angli, forgé par Bède, et, ce faisant, poussés implicitement à se ranger sous la bannière du rex Anglorum dans sa guerre contre les Danois, par ailleurs païens ${ }^{76}$. Par ce biais, la convergence est forte entre appartenance ethnique, pratique religieuse et autorité politique. Conformément aux principes développés par Bède, Alfred reforme ce principe d'unicité à son profit.

\section{EEthelstan et le Royaume des Anglais}

L'usage d'une titulature faisant référence aux Angli ne se généralisa cependant qu'à compter du règne d'Æthelstan (924-939) ${ }^{77}$. Après la

71. B. Yorke, « Anglo-Saxon gentes...»; D. N. Dumville, « Kingship, Genealogies and Regnal Lists », dans P. H. SAWYER et I. Wood éd., Early Medieval Kingship, Leeds, 1997, p. 72-104.

72. Helmut Gneuss (Handlist of Anglo-Saxon Manuscripts. A List of Manuscripts and Manuscript Fragments Written or Owned in England up to 1100, Tempe, 2001) recense $7 \mathrm{~ms}$. rédigés pendant notre période $\left(\mathrm{n}^{\circ} 75,181,410,487,555,630\right.$ et 759$)$, sans compter $3 \mathrm{~ms}$. comptant des extraits $\left(n^{\circ} 401,427\right.$ et 609$)$ et un ms. antérieur glosé pendant notre période ( $\mathrm{n}^{\circ}$ 377). Neil Ker (Catalogue of Manuscripts Containing Anglo-Saxon, Oxford, 1990) compte deux ms. de la traduction en vieil anglais pour notre période $\left(\mathrm{n}^{\circ} 32\right.$ et 180$)$, contre un ms. comptant des extraits $\left(n^{\circ} 151\right)$. Il s'agit ainsi du troisième livre non biblique le plus copié en Angleterre.

73. Ælfric of Eynsham, Sermones catholici, no 9, 1. 6-9 (éd. M. Godden, AElfric's Catholic Homilies. The Second Series Text, Londres, 1979) ; S. D. Keynes, M. Lapidge, Alfred the Great..., p. 33 ; D. WhiтеLоск, « The Old English Bede. Sir Israel Gollancz Memorial Lecture, $1962 »$, Proceedings of the British Academy, 48 (1963), p. 57-93.

74. M. Swanton, The Anglo-Saxon Chronicle, New York, 1996, p. XVIII-XIX.

75. H. Kleinschmidt, «What does the Anglo-Saxon Chronicle tell us about "ethnic origins" ? », Studi medievali, 42 (2001), p. 1-40.

76. Pour les nuances qu'impose un tel raisonnement, notamment à propos des Anglais de Lothian sous domination du roi des Écossais, voir G. Molyneaux, « Why Were Some TenthCentury English Kings... », p. 78 sq.

77. H. Hall, Studies in English Official Historical Documents, Cambridge, 1908, p. 199 ; F. M. Stenton, Anglo-Saxon England..., p. 349-353 ; S. D. Keynes, « Edgar, rex admirabilis »..., p. 6 ; S. Fоот, Ethelstan..., p. 26-28. Ce titre est totalement absent du monnayage d'Edward l'Aîné, bien que l'on ait conservé plus d'un millier de monnaies dont presque 200 sont encore lisibles. Dans les chartes d'Edward, quatre font état du titre de rex Anglorum (S 360, S 370, S 374 et S 379), mais aucune n'est au-dessus de tout soupçon 
conquête de la Northumbrie, en $926^{78}$, ce dernier se présente comme l'unique roi de tous les Anglais, ce qui se traduit par la multiplication des alliances matrimoniales avec les familles continentales les plus puissantes. En 917-919, Eadgifu, demi-sœur du roi, avait épousé Charles le Simple, roi de Francie occidentale (898-922) ${ }^{79}$, mais ces alliances se multiplient surtout après $926^{80}$. En 926, Hugues le Grand, comte de Paris (923-956), envoie une ambassade à Æthelstan et obtient en mariage sa demi-sœur, Eadhild ${ }^{81}$. La même année, Æthelstan arrange une alliance entre sa sœur, Eadgyth, et le roi scandinave d'York, Sihtric ${ }^{82}$. En 929 ou 930, Henri, roi de Francie orientale (919-936), obtient d' Æthelstan la main d'Eadgyth, sa demi-sœur, pour son fils, Otton Ir (roi en 936, puis empereur de 962 à 973) ${ }^{83}$. Quelle qu'en soit l'analyse, ces alliances signalent que le roi des Anglais, à cette date, avait capté l'essentiel de l'héritage symbolique des Carolingiens et que certaines parentèles continentales tentaient de s'en rapprocher afin de légitimer leur pouvoir croissant ${ }^{84}$. D'autres demi-sœurs du roi, dont le nom n'est pas sûr, auraient épousé d'autres princes continentaux, Conrad III, roi de Bourgogne (937-993), et/ou Louis III, roi de Provence (890-928) ${ }^{85}$. À ces alliances s'ajoutèrent les pratiques du fosterage et de la parenté rituelle. Ainsi, Louis IV d'Outremer, fils de Charles le Simple ${ }^{86}$, Hakon le Bon, fils

d'être fausse. Le plus souvent, ce roi utilise le titre de rex Angul Saxonum ou des variantes proches (19 occurrences, dont plusieurs chartes jugées authentiques) et, à une occasion, de rex occidentalium Saxonum (S 372).

78. The Anglo-Saxon Chronicle, MS D, sub anno 926 (G. CubBin éd., The Anglo-Saxon Chronicle. A Collaborative Edition, vol. VI, MS D, Oxford, 1996).

79. Ethelweard, Chronicon,p. 2 (A. Campbell éd., The Chronicle of Athelweard, Londres, 1962) ; F. M. Stenton, Anglo-Saxon England..., p. 344-345 ; S. Foot, Ethelstan ..., p. 46.

80. S. Sharp, «The West Saxon Tradition of Dynastic Marriage: with Special Reference to the Family of Edward the Elder », dans N. Higham et D. Hitl éd., Edward the Elder..., p. 79-88.

81. Æthelweard, Chronicon, p. 2 ; F. M. Stenton, Anglo-Saxon England..., p. 345 ; S. Foot, Ethelstan ..., p. 47-48.

82. The Anglo-Saxon Chronicle, MS D, sub anno 926 ; F. M. Stenton, Anglo-Saxon England..., p. 339-340. S. Foот, Ethelstan..., p. 48.

83. Æthelweard, Chronicon, p. 2 ; F. M. Stenton, Anglo-Saxon England..., p. 345-347 ; S. Foot, Ethelstan ..., p. 48-50.

84. S. McLean, «Cross-Channel Marriage and Royal Succession in the Age of Charles the Simple and Athelstan (c. 916-936) », Medieval Worlds, 2 (2015), p. 26-44 ; S. Foot, "Dynastic Strategies : The West Saxon Royal Family in Europe », dans D. Rollason, C. Leyser, H. Williams éd., England and the Continent in the Tenth Century. Studies in Honour of Wilhelm Levison (1876-1947), Turnhout, 2011, p. 237-254.

85. William of Malmesbury, Gesta Regum Anglorum, II, xxvi (éd. R. A. B. Mynors, R. Thomson, M. Winterbottom, Oxford, 1998-1999) ; S. Foot, Athelstan ..., p. 50-51.

86. Flodoard, Historia Remensis ecclesiae, sub anno 926 (S. FANNing et B. Bachrach éd., The Annals of Flodoard of Reims : 919-966, Peterborough/Plymouth, 2004) ; F. M. STENTON, Anglo-Saxon England..., p. 347 ; S. Fоoт, Ethelstan..., p. 46-47. 
du roi des Norvégiens Harald à la Belle chevelure ${ }^{87}$, et Alain Barbetorte, petit-fils du roi Alain de Bretagne ${ }^{88}$, furent accueillis à la cour.

Ce contexte se traduit par l'adoption de titres royaux de plus en plus pompeux ${ }^{89}$ et, bien souvent, par la généralisation du titre de rex Anglorum. Sur 74 chartes royales conservées, 51 y ont recours ${ }^{90}$. En additionnant les suscriptions et les souscriptions, il apparaît dans plus des deux tiers des chartes d' Æthelstan ${ }^{91}$. Après Æthelstan, ses successeurs reprirent ce titre à leur compte, au point qu'une titulature faisant référence aux Angli apparaît dans environ $80 \%$ des chartes royales jusqu'à $1066^{92}$. De même, sur les monnaies, le titre de rex Anglorum est utilisé de plus en plus couramment à compter du règne d'Eadred (946-955) et massivement à partir de celui d'Edgar (959-975). Parmi les monnaies postérieures à 954, 39\% font apparaître ce titre ou une variante ${ }^{93}$. Notons toutefois qu'Eadred (946-955) et Eadwig (955-959) reprirent parfois l'ancien usage ${ }^{94}$. Il est difficile de comprendre si cette résurgence est un choix politique ou un hasard lié à l'utilisation d'anciens " coins », si les monnaies (peu nombreuses au demeurant) sont des faux ou s'il s'agit d'un trait local, propre à un lieu de frappe spécifique.

Entre son apparition à la fin du vIII ${ }^{\mathrm{e}}$ siècle et le milieu du $\mathrm{x}^{\mathrm{e}}$ siècle, le titre de rex Anglorum a peu à peu envahi l'espace insulaire, devenant la référence la plus courante dans la pensée politique locale. Le caractère hégémonique de cette formulation dans la documentation diplomatique

87. F. M. Stenton, Anglo-Saxon England..., p. 348-349; S. Foot, Ethelstan ..., p. 54-55. 54.

88. FlodoARd, Historia Remensis ecclesiae, sub anno 919 ; S. Foot, Athelstan..., p. 53-

89. F. M. Stenton, Anglo-Saxon England..., p. 349-353 ; S. D. Keynes, The Diplomas of King Ethelred «the Unready». A Study in Their Use as Historical Evidence, Cambridge, 1980, p. 19-153.

90. S 386-390, S 393, S 395, S 399, S 400, S 402, S 403, S 405-407, S 411-416, S 418, S 418a, S 419, S 422-426, S 429-431, S 434-438, S 440-443, S 446-450, S 452-456, S 458.

91. Si l'on limite l'analyse aux chartes authentiques, cet usage concerne plus de $80 \%$ des documents : S 395-397, S 399, S 403, S 411-412, S 416-419, S 422, S 425-426, S 429-432, S 437, S 438, S 441-442, S 444, S 446-449, S 458.

92. R. DAviES, The First English Empire ...,p. 196-197. Voir A. P. SмYTH, «The Emergence of English Identity, 700-1000 », et T. Reuter, « The Making of England and Germany, 8501050 : Points of Comparison and Difference », dans A. P. Sмyтн éd., Medieval Europeans. Studies in Ethnic Identity and National Perspectives in Medieval Europe, Basingstoke, 1998, respectivement p. 24-52 et p. 53-70.

93. S. D. Keynes, « Edgar, rex admirabilis »..., p. 23 ; F. BARLow, Edward the Confessor..., p. 135-137. Sur 2036 monnaies postérieures à 946 dont l'avers est lisible, 801 recourent au titre de rex Anglorum (40\%). Si l'on exclut les 1193 cas dans lesquels le ressort n'est pas précisé, rex, c'est la solution la plus courante. Seule une monnaie d'Alfred semble frappée avec ce titre (1964.0033). Il s'agit d'un hapax.

94. Eadred sur une monnaie perdue (2009.0296) et Eadwig sur une monnaie de type N 732 (1034.0852) se présentent comme rex Zaxonum. Pour les références, nous renvoyons toujours au Corpus of Early Medieval Coin Finds et Sylloge of Coins of the British Isles... 
constituerait alors une preuve manifeste de la mise en place d'un secrétariat

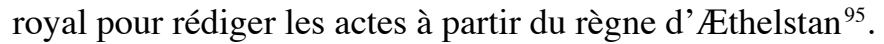

\section{Dominer les peuples anglo-saxons : les successeurs d'AEthelstan}

L'apparence d'unité que révèle l'uniformisation des titulatures dans le matériau diplomatique ne doit cependant pas mener à une approche téléologique : avant 954 , la nation anglaise n'est pas unifiée durablement ${ }^{96}$. En effet, la persistance de forts antagonismes régionaux et l'influence plus ou moins marquée des migrants scandinaves rendent complexe le paysage ethnique et identitaire du royaume.

Les « provinces multiples de la gens Anglorum ${ }^{97}$ » se font encore jour dans la documentation narrative et diplomatique. Les archevêques d'York, la communauté de St Cuthbert et d'autres institutions anglo-saxonnes du nord de l'Angleterre s'accommodèrent aisément de la présence scandinave, dont ils tirèrent profit pour s'enrichir et rester indépendants pendant la première moitié $\mathrm{du} \mathrm{x}^{\mathrm{e}}$ siècle $^{98}$. L'agenda politique des Northumbriens ne cadre donc pas avec celui des West-Saxons. Ils se soulèvent entre 941 et 944, avant d'être soumis en 946-947, de se soulever à nouveau en 948 et d'être définitivement intégrés en $954{ }^{99}$. Ces activités centrifuges amenèrent certains rois anglo-saxons à réaffirmer périodiquement leur maîtrise de la région dans leurs titulatures ${ }^{100}$. C'est le cas d'Æthelstan ${ }^{101}$, mais surtout d'Eadred (946-955) ${ }^{102}$. Après l'annexion de York, en 954, cette affirmation se fait de plus en plus rare ${ }^{103}$.

De même, la Mercie semble, dans des conditions assez confuses, avoir fait sécession pendant quelques années, à la fin du règne d'Eadwig (955-959). Tandis qu'Eadwig continuait d'exercer son autorité sur le royaume entier, c'est son jeune frère, Edgar, qui exerçait le pouvoir au nord de la Tamise. À cette occasion, Edgar reçoit le titre de rex Merciorum $^{104}$

95. S. D. Keynes, The Diplomas..., p. 39-79.

96. P. Wormald, «Engla Lond...», p. 3.

97. Wulfstan of Winchester, Vita Athewoldi, 19 (M. Lapidge et M. Winterbottom éd., Wulfstan of Winchester. Life of St Athewold, Oxford, 1996).

98. D. Hadley, The Vikings in England. Settlement, Society and Culture, Manchester, 2006.

99. The Anglo-Saxon Chronicle, MS D, à ces dates.

100. E. John, Orbis Britanniae..., p. 55.

101. S 392, sans doute un faux.

102. S 520, S 544, S 548-550, S 552a, S 569, S 572. Ces titres sont utilisés en 946, 949950 et 954, c'est-à-dire après les trois opérations militaires d'Eadred en Northumbria, en 946, 948 et 954 . Voir The Anglo-Saxon Chronicle, MS D.

103. S 633 (Eadwig), S 766 (Edgar), S 931 (Æthelred II), S 1015 (Edward le Confesseur). Voir F. BARLOw, Edward the Confessor..., p. 135-136.

104. S 667, S 675, S 676, S 676a, S 677, S 678, S 681 ; S. D. Keynes, « Edgar, rex admirabilis $» . . .$, p. 13. L'utilisation de ce titre dans deux diplômes d'Edmund et Eadred, S 514 et S 576, relève vraisemblablement de la falsification. 
et les Merciens semblent en conséquence l'avoir accepté comme roi. À la même époque, une charte originale d'Eadwig adjoint aussi à cette liste « les Saxons de l'Est et du Nord ${ }^{105} \gg[$ sic $]$.

Lorsque d'anciens royaumes sont mentionnés, hormis dans le cas des titulatures merciennes, on leur adjoint systématiquement les Angli ou les Anglo-Saxons ${ }^{106}$. Il s'agit donc toujours, d'une manière ou d'une autre, d'un pouvoir royal qui s'affirme sur plusieurs regna simultanément, même si le peuple anglo-saxon est alors présenté comme une unité. Cela pourrait justifier, aux yeux de George Molyneaux, la dimension impériale du pouvoir de ces rois, comme on en trouve trace dans le second ordo de couronnement anglo-saxon ${ }^{107}$.

\section{Edgar et la domination des peuples voisins}

La domination souvent réaffirmée, et parfois réelle, de royaumes voisins et l'intégration de populations diverses dans le royaume des Anglais témoignent d'un changement d'échelle.

Bien souvent, la titulature des chartes ajoute d'autres peuples aux peuples anglo-saxons ${ }^{108}$. Ainsi, Eadred, à la toute fin de son règne, est décrit comme « roi des Anglo-Saxons et empereur des Northumbriens, mais aussi gouverneur des païens et vainqueur des Bretons ${ }^{109}$ ». D'autres chartes parlent de « barbares » ou de « gentils ${ }^{110}$ ». Et trois cas isolés mentionnent les Cumbriens et les Écossais ${ }^{111}$, les Danois ${ }^{112}$ et les Orcadiens ${ }^{113}$. L'expansion du ressort auquel le roi destinait ses codes de loi ${ }^{114}$ et la pratique du couronnement solennel, repris à l'ordo carolingien du temps

105. S 636, probablement original.

106. S 392, S 520, S 544, S 548-550, S 552a, S 569, S 572, S 633, S 636, S 766, S 931, S 1015 .

107. G. Molyneaux, «Why Were Some Tenth-Century English Kings... », p. 63 sq.

108. Les «païens » (S 544 et S 552a) et, dans tous les autres cas, « les païens et les Bretons ».

109. S 572 : « rex Angulsexena et Norhumbre imperator paganorumque gubernator et Britonum propugnator $»$.

110. S 573 (Eadred), S 632 (Eadwig) et S 725 (Edgar).

111. S 779, qui est sans doute un faux.

112. S 1417, qui est sans doute authentique ; F. M. Stenton, Anglo-Saxon England..., p. 347-348 ; S. Foot, Athelstan..., p. 116.

113. S 914, qui est très douteuse.

114. IV Edgar 2a, § 2 (A. RoBERTSON éd., The Laws of the Kings of England from Edmund to Henry I, Cambridge, 2009) : le code s'applique aux Anglais, aux Danois et aux Bretons ; P. Wormald, The Making of English Law. King Alfred to the Twelfth Century, I, Legislation and its Limits, Oxford, 1999, p. 442-443. 
d'Æthelstan ${ }^{115}$ et perpétués par Edgar (959-975) ${ }^{116}$ ou Æthelred II (9781016) ${ }^{117}$, confirment cet aspect.

Ces titulatures semblent se nourrir de la réussite militaire des rois anglo-saxons contre leurs voisins celtiques et de la soumission de certains d'entre eux ${ }^{118}$. Parfois la soumission de roitelets insulaires est même attestée dans les listes de témoins des chartes, notamment sous Æthelstan et Edgar ${ }^{119}$. Une telle logique est particulièrement visible pendant le règne d'Edgar, notamment après les événements de 973 . En marge du concile de Chester, le roi Edgar semble avoir reçu plusieurs rois insulaires, dont ceux du Strathclyde et d'Écosse, en vue d'obtenir leur soumission, peut-être sur la Dee ${ }^{120}$. Peu avant, si l'on en croit l'analyse de Janet Nelson, reprise par George Molyneaux, le second sacre d'Edgar à Bath avait permis d'affirmer cette prétention insulaire ${ }^{121}$. Une semblable préoccupation occupe le cœur du règne d'Æthelred II, lorsque, vers 1000, il dirige une expédition pour attaquer l'île de Man et le Cumberland ${ }^{122}$. Pour autant, il est évident que ce pouvoir n'est pas intensif, mais extensif ${ }^{123}$.

115. P. Wormald, The Making..., p. 446-448 ; J. L. Nelson, « The First Use of the Second Anglo-Saxon Ordo », dans J. Barrow et A. Wareham éd., Myth, Rulership, Church and Charters, Essays in Honour of Nicholas Brooks, Aldershote, 2008, p. 117-126.

116. The Anglo-Saxon Chronicle, MS A, sub anno 973 ; F. M. Stenton, Anglo-Saxon England...,p. 368-370 ; S. D. KeYNES, «Edgar, rexadmirabilis »..., p. 48-51. Voir D. W ARNER, "Comparative Approaches to Anglo-Saxon and Ottonian Coronations », dans D. Rollason, England and the Continent..., p. 275-292.

117. Passé sous silence par le manuscrit A de la Chronique anglo-saxonne, ce fait est cité dans The Anglo-Saxon Chronicle, MS C, sub anno 979 (K. O'Brien O'KeEFFe éd., The AngloSaxon Chronicle. A Collaborative Edition, vol. V, MS C, Oxford, 2000) ; F. M. STENTon, Anglo-Saxon England..., p. 373 ; S. D. Keynes, The Diplomas..., p. 233.

118. The Anglo-Saxon Chronicle, MS D, sub annis 926 (Æthelstan) et 1031 (Cnut) ; F. M. Stenton, Anglo-Saxon England..., p. 340-342 (Æthelstan). S. Foot, Ethelstan..., p. $212 s q$; M. J. Trow, Cnut, Emperor of the North, Stroud, 2005, p. 197-198.

119. S 400, S 413 ; E. John, Orbis Britanniae..., p. 49. Voir sur ce point G. Molyneaux, «Why Were Some Tenth-Century English Kings...», p. 65 sq.

120. The Anglo-Saxon Chronicle, MS E, sub anno 972 (Edgar) (S. Irvine éd., The AngloSaxon Chronicle. A Collaborative Edition, vol. VII, MS E, Oxford, 2002). Cette situation est attestée par la présence de princes celtiques dans la liste de témoins de plusieurs chartes (S 783 et S 808), mais aussi par le témoignage tardif de Syméon de Durham (De Northymbrorum comitibus, éd. T. ArNold, Symeonis Monachi Opera Omnia, 1, Londres, 1882, p. 382-384, p. 382) ; A. Williams, « An Outing on the Dee : King Edgar at Chester, AD 973 », Mediaeval Scandinavia, 14 (2004), p. 229-243 ; C. P. LewIs, « Edgar, Chester, and the Kingdom of the Mercians, 957-9 », dans D. G. ScraGg éd., Edgar..., p. 104-123 (p. 121-122).

121. J. L. Nelson, « The Second English Ordo», dans Ead., Politics and Ritual in Early Medieval Europe, Londres, 1986, p. 361-374 (p. 369-374) ; G. Molyneaux, « Why Were Some Tenth-Century English Kings... », p. 67-68. Se référer à ce dernier article (notamment p. 68-78) pour la réalité du pouvoir exercé par les rois anglo-saxons sur les marges celtiques de l'île de Bretagne.

122. I. HowARD, The Reign of Ethelred II, King of the English, Emperor of all the Peoples of Britain, 978-1016, Oxford, 2010, p. 40-41.

123. G. Molyneaux, « Why Were Some Tenth-Century English Kings... », p. 62. 
En somme, les rois des West-Saxons incorporent dans leurs titulatures le nom d'autres peuples, souvent constitués en royaumes depuis plusieurs siècles. Ces peuples sont non seulement, depuis Alfred, les autres AngloSaxons, mais, petit à petit, des populations celtiques et scandinaves. En ce sens, le lien entre gens et regnum se distend. Dans un contexte d'expansion, les rois n'ont plus aucun intérêt à fixer une frontière qui s'arrêterait à la zone dans laquelle vivaient des Anglo-Saxons. L'utilisation d'autres ethnonymes permettait de dépasser le champ étroit de la gens comme cadre d'exercice du pouvoir, en rassemblant derrière la personne du roi d'autres groupes de personnes $^{124}$.

\section{Cnut, empereur du Nord}

Le pouvoir des rois des Anglais connut un recul brutal à la fin du $x^{\mathrm{e}}$ siècle. La mise à mort du roi Edward le Martyr (975-978) ${ }^{125}$, les débuts chaotiques du règne d' Æthelred II (978-1016) ${ }^{126}$ et la reprise des attaques vikings (vers 980) ${ }^{127}$ affaiblirent considérablement le royaume.

Après deux décennies de guerre, en 1013, le roi danois Swein à la Barbe fourchue obtint la couronne et Æthelred II se réfugia en Normandie ${ }^{128}$. À la mort de Swein, en 1014, son fils, Cnut, qui devait lui succéder, fut chassé par les Anglais. Mais, avec l'aide de son frère, alors roi des Danois, Cnut revint et, après la sanglante bataille d'Assandun (peut-être Ashingdon, Essex) et la mort du roi Edmund Côtes-de-Fer (1016), il assuma seul le pouvoir jusqu'à sa propre mort en $1035^{129}$. À la mort de son frère, Cnut se rendit au Danemark et en obtint la couronne ${ }^{130}$. Lorsque Suédois et Norvégiens commencèrent à contester le joug danois en mer Baltique, Cnut se rendit en Scandinavie pour affronter ses adversaires. Les Norvégiens,

124. S. ReYnoLDS, Kingdoms and Communities..., p. 256-331; EAD., « What Do We Mean... » (402 sq.).

125. The Anglo-Saxon Chronicle, MS A, sub anno 978 ; F. M. Stenton, Anglo-Saxon England..., p. 372-373 ; S. D. Keynes, The Diplomas..., p. 166-174 ; R. LaVelLe, Ethelred II, King of the English, 978-1016, Stroud, 2002, p. 41-44 ; A. Williams, Ethelred the Unready. The Ill-Counselled King, Londres, 2003, p. 11-14.

126. The Anglo-Saxon Chronicle, MS C, sub anno 986. S 876 et S 918 pour des déprédations sur les biens de monastères ; S. D. Keynes, The Diplomas..., p. 176-186 ; R. LAVELLE, Athelred II..., p. 44-46.

127. The Anglo-Saxon Chronicle, MS C, sub anno 980 sq. ; F. M. Stenton, Anglo-Saxon England..., p. 373 sq; R. LAVELLE, Ethelred II..., p. 47-52.

128. The Anglo-Saxon Chronicle, MS C, sub anno 1013 ; F. M. Stenton, Anglo-Saxon England..., p. 386 ; R. LAVELLE, Ethelred II..., p. 125-129.

129. The Anglo-Saxon Chronicle, MS C, sub annis 1014 et 1016 ; F. M. STEnton, AngloSaxon England..., p. 386-393 ; I. Howard, Swein Forkbeard's Invasions and the Danish Conquest of England, 991-1017, Woodbridge, 2003 ; M. Lawson, Cnut England's Viking King, Stroud, 2004, p. 25 sq., 40 sq.

130. The Anglo-Saxon Chronicle, MS E, sub anno 1019 ; F. M. Stenton, Anglo-Saxon England..., p. 401 ; M. Lawson, Cnut..., p. 88-90. 
révoltés à l'initiative du roi Olaf, furent battus en 1028 et définitivement vaincus en 1030 à la mort d'Olaf ${ }^{131}$. Enfin, désireux d'étendre son influence et de prévenir les attaques, Cnut effectua une opération militaire en Écosse, avant d'obtenir aussi la soumission d'autres potentats du monde insulaire ${ }^{132}$. En ce sens, Cnut a dominé un ensemble de peuples : Anglais, Danois, Norvégiens, Suédois, et peut-être Écossais, Gallois, Irlandais. Aussi bien par ses dimensions que par la diversité de ces regna, le pouvoir de Cnut était impérial ${ }^{133}$, et c'est d'ailleurs le titre que lui donne l'Encomiaste : « quinque regnorum imperator ${ }^{134} »$. Le rôle que joue la conquête militaire dans la construction de cet empire transmaritime, thalassocratique, fait d'ailleurs penser à l'idée d'un empire jailli des confins et prenant le contrôle d'une zone nourricière ${ }^{135}$.

Les titulatures de Cnut laissent parfois apparaître la dimension pluriethnique de son empire : " roi d'Angleterre et du Danemark [et de Norvège] ${ }^{136} »$. Néanmoins, tous les documents en question sont douteux ou issus de copies tardives. Contrairement à ses prédécesseurs, Cnut n'a visiblement pas particulièrement insisté sur le caractère multiethnique de son pouvoir, mettant plutôt en lumière son attachement à l'Angleterre afin de sécuriser le pouvoir qu'il y avait acquis. Par contraste, sa domination sur les autres gentes passe par des formes de délégation d'autorité à des proches, plus ou moins fiables, sur le modèle carolingien ${ }^{137}$. Ainsi, le Danemark est confié vers 1023 à Thorkell ${ }^{138}$, puis à Harthacnut, fils du roi, en 1026 ; en Norvège, sa première femme, Ælfgifu de Northampton, et leur fils, Swein, tiennent le pouvoir entre 1030 et $1035^{139}$. De même, l'adoption par Cnut de l'orbe et d'une nouvelle couronne, sur le modèle de celle d'Henri II, après la conquête de la Norvège témoigne de prétentions

131. The Anglo-Saxon Chronicle, MS E, sub annis 1025 et 1028 ; F. M. Stenton, AngloSaxon England..., p. 402-405 ; M. Lawson, Cnut..., p. 94-98.

132. The Anglo-Saxon Chronicle, MS E, sub anno 1031 ; F. M. Stenton, Anglo-Saxon England..., p. 419 ; M. Lawson, Cnut..., p. 100-104.

133. P. H. SAwYeR, "Cnut's Scandinavian Empire », dans A. Rumble éd., The Reign of Cnut. King of England, Denmark and Norway, Londres, 1994, p. 10-26 ; T. Bolton, The Empire of Cnut The Great. Conquest and the Consolidation of Power in Northern Europe in the Early Eleventh Century, Leyde, 2009.

134. Encomium Emmae Reginae, A. CAmpbell éd., Encomium Emmae Reginae, Cambridge, 1998, p. 34.

135. G. Martinez-Gros, Brève histoire des empires....

136. S 965. S 976. I Cnut, prologus (A. ROBERTSOn éd., The Laws...).

137. G. BüHRER-ThierRY, "Centres et périphéries dans l'empire carolingien : de la conception à la construction de l'empire », dans F. Hurlet éd., Les Empires..., p. 145-155 (p. 150).

138. The Anglo-Saxon Chronicle, MS C, sub anno 1023 ; F. M. Stenton, Anglo-Saxon England..., p. 401-102 ; M. Lawson, Cnut..., p. 92.

139. F. M. Stenton, Anglo-Saxon England..., p. 404-406 ; M. Lawson, Cnut..., p. 98-100, p. 104-105 et p. 123-124. 
impériales ${ }^{140}$. Ses déplacements, en particulier à Rome en 1027 pour le couronnement impérial de Conrad II, le mariage entre sa fille Gunnhild et le futur empereur Henri III en $1035^{141}$, et la constitution d'un personnel curial, issu du Danemark et d'Angleterre ${ }^{142}$, montrent la dimension européenne de son pouvoir et la dimension impériale de son règne.

\section{La titulature impériale des rois des Anglais}

Le ressort inclus dans les différentes titulatures à compter du règne d' Æthelstan montre un potentiel impérial, fondé sur la domination de plusieurs royaumes, dont certains souverains ne se saisissent pas (Alfred, Cnut) et sur lequel d'autres insistent (Æthelstan, Edgar). Il reste donc à poser la question du titre lui-même, mais aussi de l'invocation utilisée par les souverains.

Rector, primicerius, imperator et basileus

Le titre que les rois anglo-saxons utilisaient aux $\mathrm{X}^{\mathrm{e}}-\mathrm{XI}^{\mathrm{e}}$ siècles témoignait également de leur ancrage dans l'imaginaire impérial. Si le titre de rex ou cyning est le plus courant ${ }^{143}$, il est, à certains moments, supplanté par basileus $^{144}$ et imperator ${ }^{145}$. Entre le règne d'Alfred et celui d'Harold II Godwineson, 191 chartes recourent à l'un, à l'autre ou à ces deux titres, soit un peu plus d'un quart des documents (que l'on regarde les seuls documents authentiques ou la totalité des actes conservés). Parmi ces deux titres toutefois, imperator est nettement plus rare. Seuls quelques documents authentiques de la seconde moitié du $\mathrm{x}^{\mathrm{e}}$ siècle sont préservés ${ }^{146}$.

Si l'on met de côté un acte douteux d'Alfred ${ }^{147}$, ces titres apparaissent sous le règne d'Æthelstan. Représentant environ $10 \%$ des actes conservés chez la plupart des souverains (Æthelstan, Edmund, Eadred, Eadwig, Cnut,

140. P. E. Schramm, Sphaira. Globus. Reichsapfel. Wanderung und Wandlung eines Herrschaftszeichens von Caesar bis zu Elisabeth II. Ein Beitrag zum "Nachleben" der Antike, Stuttgart, 1958 ; C. E. Karkov, The Ruler Portraits of Anglo-Saxon England, Woodbridge, 2004, p. 126-128.

141. F. M. Stenton, Anglo-Saxon England ..., p. 406-408 ; M. Lawson, Cnut..., p. 99-100 et p. 104-105.

142. S. D. Keynes, « Cnut earls », dans A. Rumble éd., The Reign of Cnut..., p. 43-88.

143. Toutes les monnaies dont l'avers est lisible utilisent ce titre, à trois exceptions près, puisque trois monnaies d'Eadred utilisent le titre de monarchus (1034.0643, 1034.0644, 1034.0668). Quant aux chartes, sur 460 jugées fiables, 293 utilisent le mot rex dans la suscription $(64 \%)$ et 370 dans la souscription ( $80 \%)$.

144. 175 occurrences $(24 \%) .91$ dans des chartes fiables $(23 \%)$.

145. 21 occurrences ( $3 \%) .7$ dans des chartes fiables $(2 \%)$.

146. S 572, S 775, S 865 , S 869, S 874, S 886, S 903. Les cinq dernières chartes ont été émises par Æthelred II.

147. S 351. 
Edward le Confesseur), imperator connaît une vogue exceptionnelle sous le règne d'Edgar (roi en 959-975, 75\%) et d' Æthelred II (roi en 978-1016, $44 \%)^{148}$. Une nouvelle fois, le renouveau remonte aux années 920 , avec les campagnes victorieuses d'Æthelstan, mais se renforce ultérieurement, pendant le règne d'Edgar, au moment où le royaume des Anglais est florissant ${ }^{149}$. Dans les Translatio et Miracula S. Swithuni, Lantferth signale également qu'Edgar est basileus insignus atque inuictissimus, prepotens ac clementissimus necnon gloriosissimus ${ }^{150}$. À son maximum dans les années 980, l'emploi de ce titre recule avec le second âge viking, mais refait surface au moment où Cnut met en place l'empire du Nord. Bien que l'usage recule rapidement après le règne de Cnut, même Edward le Confesseur y a recours, notamment sur ses sceaux ${ }^{151}$, ce qui laisse à penser que cet usage était profondément ancré dans l'entourage du roi.

Les Anglo-Saxons utilisaient aussi massivement le titre de primicerius. Si le titre semble apparaître à nouveau sous le règne d' Æthelstan ${ }^{152}$, l'usage se généralise en réalité dans les années 950 , primicerius est majoritairement utilisé sous Eadred et Edgar, avec 57 occurrences ( $8 \%$ de leur production) ${ }^{153}$. Néanmoins, il disparait tout aussi rapidement, avec seulement trois occurrences dans des documents authentiques postérieurs au règne d'Edgar. De même, le titre de rector est courant dans les chartes ${ }^{154}$, principalement pendant le règne d'Eadred ${ }^{155}$.

Les titres de basileus et imperator ont été l'objet de nombreux débats parmi les spécialistes. Richard Drögereit et Henry Loyn estimaient qu'imperator n'apparaissait que dans des documents douteux et que basileus, conformément à son sens en grec ancien, était un simple

148. Un calcul portant sur les seuls documents authentiques permet de nuancer cette hégémonie des années 960 . Toutefois, Edgar et Æthelred II arrivent toujours en tête (43 et $45 \%$ ), devant Æthelstan et Cnut (28\% et $31 \%$ ).

149. H. Hall, Studies.... p. 201 ; J. Campbell, The Anglo-Saxon State..., p. 40-41.

150. LantFerth, Translatio et miracula S. Swithuni, Praefatio (M. LAPIDGe éd., The Cult of St Swithun, Oxford, 2003). Ces titres, présents dans la titulature de certains rois mérovingiens et carolingiens, ont pu être transmis par le biais de collections canoniques diverses.

151. F. BarLow, Edward the Confessor..., p. 135-137. Lynn Jones note qu'Edward le Confesseur disposait de sceaux à deux faces, comme à Byzance, à rebours de l'usage continental des sceaux à une face («From Anglorum basileus to Norman Saint: the Transformation of Edward the Confessor », Haskins Society Journal, 12 (2002), p. 99-120, ici p. 103-105). Voir aussi sur ce point C. Morrisson, « Anglo-byzantina. Monnaies et sceaux des $\mathrm{IX}^{\mathrm{e}}$-XIIII ${ }^{\mathrm{e}}$ siècles outre-Manche », dans Mélanges dédiés à Jean-Claude Cheynet, Paris, 2018, p. 471-485.

152. S 408 et $\mathrm{S} 410$ sont très douteuses.

153. S 505, S 555, S 560, S 561, S 563, S 564, S 568, S 570, S 617, S 618, S 676, S 676a, S 678, S 708, S 726, S 735, S 743, S 748, S 773, S 785, S 790, S 791, S 794a, S 802, S 803, S 884, S 905, S 921 .

154. 122 occurrences $(16 \%)$. Parmi les chartes authentiques, on le retrouve 84 fois (22\%).

$155.66 \%$ des chartes, qu'elles soient authentiques ou non. 
synonyme de rex ${ }^{156}$. Toutefois, outre les sept chartes authentiques utilisant imperator, Eric John a souligné dès 1966 que Byrhtferth de Ramsey, dans la Vita Oswaldi rédigée autour de l'an mil, nommait lui aussi Edgar imperator, et même imperator totius Albionis ${ }^{157}$. Or, comme le signale Gilbert Dagron à propos de basileus, tous ces termes sont « bifrons » : renvoyant étymologiquement à la royauté ou à des fonctions militaires, ils sont attachés au nom des empereurs, présents ou passés, que ce soit à Rome, à Byzance ou à Aix-la-Chapelle ${ }^{158}$. Basileus, qui entrait dans le titre des empereurs d'Orient depuis Héraclius ${ }^{159}$, put être introduit en Angleterre par deux canaux : les textes d'auteurs continentaux friands d'hellénismes et passés par l'Angleterre, comme Fredegaud de Brioude et Abbon de Fleury ${ }^{160}$, et la circulation assez clairement attestée de mercenaires scandinaves et d'exilés grecs entre Byzance et le monde insulaire ${ }^{161}$. Primicerius, courant dans le Codex Theodosianus ${ }^{162}$, était encore commun à Byzance à la période étudiée pour désigner un chef de bureau de l'administration ou un chef

156. R. DRÖGEREIT, « Kaiseridee under Kaisertitel bei den Angelsachsen », Zeitschrift der Savigny-Stiftung für Rechtsgeschichte, Germanistische Abteilung, 69 (1952), p. 24$73 ; \mathrm{H}$. Loyn, « The Imperial Style of the 10th Century Anglo-Saxon Kings », History, 40 (1955), p. 111-115. Ils auraient également pu signaler qu'imperator renvoyait à un sens romain classique : " chef des armées », " général victorieux ». C'est d'ailleurs ce que fait George Molyneaux dans son récent article à ce sujet («Why Were Some Tenth-Century English Kings... », p. 63), en considérant ces traits lexicaux comme une résurgence du style herméneutique qui se développe en Angleterre à ce moment.

157. ByrhtFerth of Ramsey, Vita Oswaldi, IV, 6 et 17 (M. Lapidge éd., The Lives of St Oswald and St Ecgwine, Oxford, 2009) ; E. JoHn, Orbis Britanniae..., p. 52-53. Pour la datation, voir M. LAPIDGE, The Lives of St Oswald ..., p. LXVII-LXVIII.

158. G. DAGRON, «Empires royaux... », p. 82.

159. J. ShePARD, « From the Bosporus to the British Isles : the Way from the Greeks to the Varangians », dans Drevnejshie gosudarstva vostochnoi Evropy, Moscou, 2010, p. 15-42 (p. 23).

160. M. Lapidge, « The Hermeneutic Style in Tenth-Century Anglo-Latin Literature », Anglo-Saxon England, 4 (1975), p. 67-111 (p. 72 et p. 98). Notons également l'existence d'au moins une liste d'empereurs, carolingiens et byzantins, dans le ms. London, BL, Cotton Vespasian B.vi, copié à Saint-Denis pendant le règne de Louis le Pieux et introduit en Angleterre pendant le règne d' $Æ$ thelred II (H. Gneuss, Handlist of Anglo-Saxon Manuscripts. A List of Manuscripts and Manuscript Fragments Written or Owned in England up to 1100, Tempe, 2001, p. 70).

161. J. ShePARD, « From the Bosporus to the British Isles... », notamment p. 23-32 ; M. LAPIDGE, «Byzantium, Rome and England in the Early Middle Ages », dans Roma fra oriente et occidente. Settimane di studio del centro italiano di studi sull' alto medioevo XLIV, Spolète, 2002, p. 363-400.

162. Il s'agit d'un titre du Codex : voir Codex Theodosianus, I, 33 (T. Mommsen, R. Delmaire, P. Krueger, P. M. Meyer, J. Rougé, Code Théodosien. I-XV, Code Justinien, constitutions sirmondiennes, Paris, 2008). On en compte une trentaine d'occurrences dans le texte. Sur l'importance du code théodosien au Moyen Âge et, en particulier, en Angleterre, voir P. Wormald, The Making..., I, p. 36-39 et p. 282 (pour son influence sur le code d'Alfred). 
de la garde impériale (et notamment un chef de la garde varangienne) ${ }^{163}$. Imperator et rector sont pour leur part connus des auteurs anglo-saxons depuis les Dark Ages, mais ont sans doute été utilisés par mimétisme des traditions diplomatiques et législatives carolingiennes ${ }^{164}$. En ce sens, il est peu probable que la chancellerie des rois les plus puissants de l'ère anglosaxonne tardive ait utilisé des termes aussi connotés sans arrière-pensée ${ }^{165}$, même si Jonathan Shepard estime qu'un tel usage oscille entre connotation impériale et volonté de prendre un titre susceptible d'inspirer le respect aux anciens Varègues installés en Angleterre ${ }^{166}$.

S'ils étaient impériaux, comment interpréter ces titres ? Freeman et Stubbs pensaient qu'ils étaient utilisés pour affirmer l'indépendance de l'Angleterre face au pouvoir montant des Ottoniens en Germanie et comme une manière de renforcer les revendications hégémoniques du roi à l'encontre des princes bretons insulaires ${ }^{167}$. L'hypothèse d'une compétition avec les princes allemands semble plutôt ressortir aux préoccupations des Anglais dans le second $\mathrm{XIX}^{\mathrm{e}}$ siècle ${ }^{168}$. En revanche, la dimension hégémonique de leur pouvoir sur le monde insulaire, la remise au goût du jour du vieux titre de bretwalda et le droit qu'il offrait en matière d'ingérence dans les affaires politiques et ecclésiastiques des princes celtes correspondent parfaitement avec l'agenda diplomatique des rois de Winchester ${ }^{169}$. L'image que les rois ont tenté de convoyer dans les quelques sources graphiques qui nous sont parvenues confirment cette ambition impériale ${ }^{170}$.

163. A. Kazhdan, « Primikerios », dans A. Kazhdan éd., The Oxford Dictionary of Byzantium, t. III, Oxford, 1991, p. 1719-1720.

164. J. Nelson, « Kingship and Royal Government », dans R. McKitterick éd., The New Cambridge Medieval History, vol. II, c. 700-c. 900, Cambridge, 1995, p. 383-430 (p. 424). A minima les capitulaires carolingiens étaient très bien connus en Angleterre. Voir H. GNEuss, Handlist of Anglo-Saxon Manuscripts..., passim.

165. Pour Ian Howard (The Reign of Ethelred II..., p. 18), Edgar, en utilisant basileus comme titre se rend égal aux dirigeants de l'Empire romain d'Orient. Patrick Wormald (The Making..., p. 444-445) affirme que la « posture impériale était consciemment cultivée » par les rois lorsqu'ils utilisaient des termes faisant si explicitement référence à l'histoire de Rome, de Constantinople ou de l'Europe carolingienne.

166. J. SHePARD, « From the Bosporus to the British Isles... », notamment p. 23 et p. 25. Ce qu'il dit à propos de basileus vaut donc aussi pour primicerius.

167. E. Freeman, The History of the Norman Conquest of England, Oxford, 1867, t. 1, p. 133-147 et p. 548-565 ; W. StubBs, The Constitutional History of England, Oxford, 1874, t. 1 , p. 177.

168. C'est néanmoins une analyse à laquelle semble souscrire George Molyneaux («Why Were Some Tenth-Century English Kings... », p. 68).

169. F. M. Stenton, Anglo-Saxon England..., p. 34 ; E. John, Orbis Britanniae..., p. 5-6, 15-18, 50-52 ; J. L. Nelson, Politics and Ritual..., p. 302-303 ; F. Barlow, Edward the Confessor..., p. 137. Le titre de brytenwalda est d'ailleurs utilisé dans la charte S 427.

170. C. Karkov, The Ruler Portraits of Anglo-Saxon England..., p. 66-67. 


\section{Dominer un territoire pour incorporer plusieurs regna}

L'unification de l'Angleterre est, on l'a vu, une construction idéelle reposant avant tout sur l'implication de la papauté et la mobilisation du mythe, structuré par Bède, des Anglais comme nouveau peuple élu ${ }^{171}$. Mais l'Angelcynn, la gens Anglorum, est aussi associée à une terre promise, Englaland $^{172}$. Cet interstice entre titulature incluant un peuple et titulature incluant un territoire permet plus facilement encore l'inclusion dans le regnum de populations hétérogènes.

Cette dynamique peut être observée dès le règne d'Alfred. Son biographe, Asser, lui attribue le titre d'Anglorum Saxonum rex, comme dans les chartes, maisily adjoint une revendication plus large: «omnium Britanniae insulae Christianorum rector ${ }^{173} »$. Si la nature du pouvoir (rector) renvoie sans doute à un ministerium eschatologique sur le modèle carolingien, le lieu de son exercice est désormais un espace qui coïncide avec les frontières de l'île de Bretagne. À la suite d'Alfred, les rois adoptent souvent dans leurs chartes une titulature qui renvoie à une telle zone géographique, comme Britannia $^{174}$ et Albio $^{175}$. Il existe quelques documents antérieurs aux années 920 utilisant ces termes ${ }^{176}$, mais ils semblent très douteux. En conséquence, il est fort à parier que ces titulatures apparurent sous le règne d' Ethelstan ${ }^{177}$. Leur usage massif n'est repérable que sous son règne et celui de son neveu, Edgar $^{178}$, à l'époque de la réforme bénédictine, au moment où les œuvres de

171. P. Wormald, « Engla Lond... », p. 13-14 ; R. Davies, The First English Empire..., p. $199-200$.

172. S. Fоoт, «The Making of Angelcynn : English Identity before the Norman Conquest », Transactions of the Royal Historical Society, 6 (1996), p. 25-49 (p. 32). Le titre de Englalandes cyncg apparaît dans une charte d'Edward le Confesseur (S 1047), mais il s'agit vraisemblablement d'un faux. Voir F. BARLow, Edward the Confessor..., p. 135-137.

173. Asser, Vita Alfredi, 1.

174. 214 occurrences. Parmi les chartes authentiques, un peu plus de $20 \%$ des cas.

175. 137 occurrences. Parmi les chartes authentiques, un peu plus de $15 \%$ des cas.

176. Sous Offa, la charte S 155 utilise ce terme. Sous Æthelstan, les chartes S 351 et S 379 l'utilisent aussi. Toutes ces chartes sont très douteuses.

177. E. John, Orbis Britanniae ..., p. 49 ; S. Fоот, Athelstan ..., p. 226. Albio (S 411, S 432 et S 437) et Britannia (S 407, S 412-413, S 416-419, S 422, S 425-426, S 429-431, S 438, $\mathrm{S}$ 444-449 et $\mathrm{S} 458$ ). L'utilisation de ces termes fait pencher la balance vers la fin du règne, dans tous les cas. Les chartes en italiques sont des originaux probables. Dans le monnayage d'Æthelstan, le terme est très courant. Sur les 289 monnaies préservées dont l'avers est lisible, Æthelstan porte 95 fois le titre de rex totius Brittaniae et une fois celui de rex Britanniae, ce qui représente un tiers de sa production. De nombreux ateliers et de nombreux monétaires sont représentés dans l'échantillon préservé.

178. Dans respectivement $69 \%$ et $78 \%$ de ses chartes préservées. Par contraste, ce taux est proche ou inférieur à $40 \%$ chez Æthelred II, Cnut, Eadred et Edward le Confesseur. C'est aussi le point de vue de Simon Keynes et de Julia Crick à propos des chartes dites « Dunstan B » : S. D. KeYnes, « Edgar, rex admirabilis »..., p. 7, $26-27$; J. CRICK, « Edgar, Albion and Insular Dominion », dans D. SCRAGG éd., Edgar..., p. 158-170 (p. 164-165). Pour 
Bède furent massivement redécouvertes dans les milieux ecclésiastiques ${ }^{179}$. On retrouve même ce type de titre dans des sources narratives écrites dans ces mêmes milieux. Vers 972-973, Lantfred de Fleury/Lantferth de Winchester, dans les Translatio et Miracula S. Swithuni, parle d'une époque «Eadgaro regnante $[\ldots]$ gentibus imperante $[\ldots]$ commorantibus diffuse in insula quae Albio nuncupata dicitur ${ }^{180} »$. Dans l'Epitomé de cette même vie, dans les années 980-990, Flfric d'Eynsham décrit Edgar comme « totius Albionis insule imperator ${ }^{181} »$. Dans son Chronicon, composé entre 975 et 983 , Æthelweard en fait le «monarchus Brittannum ${ }^{182} »$. Mieux encore, dans le sacramentaire de Ratold, qui contient le second ordo anglo-saxon sous une forme hybride, le titre donné au roi est celui de « rex totius Albionis $^{183}{ }$. Le titre de rex [totius] Brittaniae est également utilisé sur les monnaies de l'époque du roi Edgar ${ }^{184}$.

Aussi bien Britannia qu'Albio sont des références classiques héritées de la géographie grecque (Ptolémée) et romaine (Pline), mais introduites en

ces chartes, voir S. D. Keynes, « The "Dunstan B" charters », Anglo-Saxon England, 23 (1994), p. 165-193.

179. J. CRICK, « Edgar, Albion... », p. 163-165 ; R. Davies, The First English Empire..., p. 196.

180. Lantrerth, Translatio et miracula S. Swithuni, Praefatio. Pour la datation, voir M. LAPIDGE, The Cult of St Swithun..., p. 236.

181. Ælfric of Eynsham, Epitome de S. Swithuni, 1 (M. Lapidge éd., The Cult of St Swithun...). Pour la datation, voir p. 558.

182. Æthelweard, Chronicon, IV, 9. Pour la datation, voir A. CAmpbell, The Chronicle..., p. XIII.

183. Ratold de CoRbie, Sacramentaire (N. ORchard éd., The Sacramentary of Ratoldus [Paris, BNF, lat. 12052], Londres, 2005, p. 49). Les opinions divergent quant à savoir s'il date de l'époque d'Edward l'Aîné ou Æthelstan ou de l'époque d'Edgar. Pour la première hypothèse, voir J. L. Nelson, « The Second English Ordo »..., p. 365-366. Pour la seconde hypothèse, voir C. Hohler, "Some Service-Books of the Later Saxon Church », dans D. Parsons éd., Tenth-Century Studies. Essays in Commemoration of the Millennium of the Council of Winchester and the Regularis Concordia, Chichester, 1975, p. 60-83 (p. 68). Le débat sur la datation n'a pas vraiment d'incidence sur notre propos, puisque notre analyse pointe l'importance des règnes d'Æthelstan et d'Edgar dans l'affirmation d'un discours hégémonique. Pour une analyse plus complète, voir J. CRICK, «Edgar, Albion... », p. 165.

184. S. D. KEYNES, «Edgar, rex admirabilis »..., p. 23-24. On compte, sur 2793 monnaies postérieures au règne d'Æthelstan et dont l'avers est lisible, un nombre limité de cas (un peu moins de $2 \%$ ) : 14 pour le titre de rex totius Britanniae et 32 pour celui de rex Britanniae. La quasi-totalité des monnaies utilisant le premier titre appartient au monnayage d'Edgar antérieur à 973 (12), les deux autres appartenant au monnayage d'Eadwig (1034.0791, 1034.0780). Le titre de rex Britanniae est porté aussi bien par Edmund que par Edgar. Néanmoins, les monnaies d'Edmund sont très abîmées et il est difficile d'être certain de cette interprétation. Ces monnaies ont, pour beaucoup, été frappées dans le nord du royaume, à Chester (5), Derby (7 à coup sûr et sans doute 2 de plus), Tamworth (2) ou York (10). Ce localisme dans la frappe n'est pas sans évoquer la volonté d'afficher une main mise sur les régions périphériques du royaume, mais sans doute aussi sur les voisins gallois et écossais d'Edgar. Notons que ces monnaies relèvent de types variés et ont été frappées par de nombreux monétaires. 
Angleterre par Bède ${ }^{185}$. Elles servirent donc d'unités territoriales préétablies susceptibles de légitimer les revendications des rois ${ }^{186}$. La tendance hégémonique d'Æthelstan et de certains de ses successeurs, comme Edgar ou Cnut, s'en trouva renforcée et justifiée ${ }^{187}$, tandis que les rois en question gagnaient en majesté ${ }^{188}$. Dans certains cas spécifiques, Albio et Britannia sont spécifiquement définies comme étant une unité d'ordre territorial et géographique : une insula ${ }^{189}$, une prouincia ${ }^{190}$, un tellus ${ }^{191}$, une regio ${ }^{192}$ ou un rus $^{193}$. Parfois, d'autres îles, régions ou provinces voisines sont ajoutées ${ }^{194}$. Force est de constater que ces formules se multiplient après le règne d'Eadred et deviennent presque communes sous les règnes d'Eadwig, Edgar, Æthelred II et Cnut ${ }^{195}$.

Dans la mesure où les rois de Winchester dominaient divers peuples, initialement gouvernés par plusieurs rois, qu'ils nourrissaient une dynamique expansionniste et tentaient d'incorporer les nouvelles populations tout en maintenant des formes de différentiation entre elles, nous avons affaire à des discours de nature impériale.

\section{La mission eschatologique du roi}

L'ambition insulaire démontrée par le recours aux termes Britannia et Albio se trouve redoublée, dans la littérature anglo-saxonne, par celle des archevêques de Canterbury qui revendiquent une primatie sur le même espace $^{196}$. Ainsi, la Vita S. Dunstani du clerc B., écrite à la toute fin du

185. Les Bretwaldas dominaient parfois la totalité de l'île de Britannia et certaines îles voisines : Bède le VéNÉrAble, Historia ecclesiastica, II, v ; J. CRICK, « Edgar, Albion... », p. 166.

186. R. Davies, The First English Empire..., p. 35-36. Julia Crick signale la possibilité d'une compétition avec les rois des Ecossais, qui revendiquaient un titre semblable, en tant que maître du royaume d'Alba : J. CRICK, « Edgar, Albion... », p. 168-169.

187. E. John, Orbis Britanniae..., p. 59-62 ; S. Foot, Ethelstan..., p. 213 ; R. Davies, The First English Empire..., p. 8-9.

188. E. JoHn, Orbis Britanniae ..., p. 60 ; S. D. KeYNES, « Edgar, rex admirabilis ».., p. 7 ;

R. DAVIES, The First English Empire ..., p. 49-50 ; J. CRICK, « Edgar, Albion... », p. 164-165.

189. S 388, S 420, S 427, S 433, S 573, S 581, S 591, S 598, S 609, S 615, S 617, S 632, S 669, S 697, S 724-725, S 736, S 779, S 782, S 797, S 816, S 827, S 838, S 866, S 874, S 898, S 904, S 933, S 938, S 940, S 942, S 969, S 980. Les chartes en italiques sont authentiques.

190. S 498, $S 629, \mathrm{~S} 681$.

191. S 583, S 595, S 596, S 602, S 609, S 620, S 622, S 624, S 638, S 641, S 663, S 712, $S 755, S 763, \mathrm{~S} 862, \mathrm{~S} 879, \mathrm{~S} 900, \mathrm{~S} 923, \mathrm{~S} 955, \mathrm{~S} 1003, \mathrm{~S} 1031$.

192. $S 695, \mathrm{~S} 756, \mathrm{~S} 761, S 850, \mathrm{~S} 953$.

193. S 498, S 565, S 670, S 675, S 676, S 676a, $S 678$.

194. S 353, S 404, S 470, S 544, S 552a, S 731, S 779, S 796-797, S 845, S 904, S 931, S 959, S 976, S 1012.

195. Avant comme après ces règnes, le pourcentage de ces formules dans la documentation est inférieur à $10 \%$. Elle est supérieure sous Æthelred II (14\%), Eadwig, Edgar et Cnut $(16 \%)$.

196. J. CRICK, «Edgar, Albion... », p. 164. 
$\mathrm{X}^{\mathrm{e}}$ siècle, regroupe toute la population insulaire sous le terme de «populus Albionum », comme pour mieux les placer derrière l'archevêque Augustin de Canterbury ${ }^{197}$. De la sorte, on observe, en particulier dans le second $\mathrm{x}^{\mathrm{e}}$ siècle, un usage commun pour parler du roi et de l'archevêque, en leur attribuant un ressort qui regroupe la totalité des insulaires.

L'alliance objective entre ces deux figures renvoie à la mission eschatologique du souverain envers ses sujets. Cela rappelle les formes de « césaro-papisme » byzantin ou de «théocratie royale » carolingienne ${ }^{198}$. Asser, quand il parle d'Alfred, le nomme de fait protecteur des autres peuples de Bretagne, mais à condition qu'ils soient chrétiens : « omnium Britanniae insulae Christianorum rector ${ }^{199} »$. L'utilisation des titres carolingiens comme rector et gubernator permet de rendre sensible ce ministerium. L'absence de titres semblables à celui proposé par Asser a tendance à en montrer les limites, sinon e silentio $^{200}$.

Ce rôle eschatologique s'accompagne d'une forme de sacralité du monarque. L'utilisation d'invocations plus ou moins complexe permet de construire cette image ${ }^{201}$. Elle est attestée dès le règne d'Alfred ${ }^{202}$, mais se généralise surtout sous Æthelstan (dans $80 \%$ de ses chartes authentiques). Par la suite, cet usage se développe massivement de la fin du règne d'Edgar jusqu'au début de celui d'Æthelred II, avant de connaître un net recul jusqu'au règne d'Edward le Confesseur. Sous une autre forme, dans les 57 chartes originales, seules 4 n'utilisent pas d'invocation initiale, toutes les autres débutant par un chrisme, un «alpha oméga », un signum et/ou une invocation verbale développée ${ }^{203}$. L'invocation du nom divin comme

197. B., Vita Dunstani, 2 (M. Lapidge et M. Winterbottom éd., The Early Lives of Dunstan, Oxford, 2012). Pour la datation, voir p. LXIV.

198. Pour une discussion de ces aspects dans le contexte byzantin et carolingien, voir G. DAGRON, Empereur et prêtre. Étude sur le « césaropapisme » byzantin, Paris, 1996 ; A. Boureau, « Des politiques tirées de l'Écriture. Byzance et l'Occident », Annales HSS, 55 (2000), p. 879-887 (p. 883). Pour une discussion de ces aspects dans le contexte insulaire, voir E. John, Orbis Britanniae..., p. 60 ; B. SNOOK, « Bishops and Pawns : Parallels between "Caesaropapism" and Crusade Ideology in Tenth-Century England and Thirteenth-Century Denmark », Quaestio Insularis, 8 (2007), p. 151-178.

199. Asser, Vita Alfredi, 1.

200. Dans les titulatures préservées dans le matériau diplomatique, aucun roi n'est à proprement parler gubernator, rector ou defensor Christianorum. Seules quelques chartes font références à une relation aux païens, soit avec un roi présenté comme leur opposant (propugnator paganorum, S 931 sous Æthelred II), soit plus étrangement comme leur protecteur (gubernator paganorum, S 569 et S 572 sous Eadred, S 633 sous Eadwig) ou roi (rex... progenie paganorum, S 766 sous Edgar). Aucune de ces chartes n'est au-dessus de tout soupçon. Les dates de conservation des manuscrits et les monastères ayant conservé l'archive sont très variables, allant du $\mathrm{XI}^{\mathrm{e}}$ siècle au XIV ${ }^{\mathrm{e}}$ siècle et de Wilton à Burton.

201. 355 occurrences (48 \%). Dans les chartes authentiques, 175 occurrences (44\%).

202. S 350 est authentique.

203. Parmi les originaux, 14 utilisent une invocation verbale (S 624, S 646, S 736, S 738, S 801, S 884, S 892, S 898, S 916, S 974, S 994, S 1008, S 1394 et S 1407), une s'ouvre sur 
soutien légitimant l'autorité royale transforme le souverain en agent de la volonté divine sur terre. En conséquence, la dimension sacrée de sa fonction est clairement renforcée.

\section{La qualité de l'emprise : universalisme et expansionnisme}

La façon de présenter le ressort du roi entre également en ligne de compte dans ses prétentions impériales. Le ministerium eschatologique du roi donne à son pouvoir une vocation universelle et peut se traduire par un discours expansionniste.

Une des façons de rendre compte du caractère universel du pouvoir est d'utiliser l'adjectif totius dans le ressort. Le roi n'est pas seulement rex Albionis, il est, très souvent, rex totius Albionis. L'usage de totius est extrêmement courant dans les chartes ${ }^{204}$. Néanmoins, il distingue particulièrement le règne d' Æthelstan (59\%), d'Edgar (44\%), d' Æthelred II (35\%), de Cnut (23\%) et d'Edward le Confesseur (35\%). La fin du règne d' Æthelstan, les débuts de celui de d'Æthelred II et Cnut présentent les valeurs les plus fortes (plus de la moitié des actes, authentiques ou non). L'utilisation de totius ne se limite pas aux chartes, puisqu'on le retrouve aussi dans de très nombreuses monnaies à compter du règne d' $Æ$ thelstan ${ }^{205}$. En ce sens, l'hypothèse impériale s'appuie sur une hégémonie insulaire dûment affirmée par les souverains les plus marquants de l'époque ${ }^{206}$. Une autre façon de montrer que le pouvoir du roi s'étend au-delà des bornes de son royaume passe par l'usage du terme orbis $^{207}$. Même si le terme est présent à l'état de trace, son usage semble attesté et il prend une nouvelle fois son essor sous le règne d' Æthelstan pour perdurer jusqu'au début de celui d' Æthelred II. Ces deux termes renvoient à la prétention universelle des pouvoirs de type impérial.

Une autre manière d'indiquer le pouvoir persistant, quoique lâche, du roi en-dehors de ses frontières, consiste à indiquer qu'il était aussi le

un « alpha oméga » (S 736), 33 portent un chrisme (S 624, S 636, S 646, S 649, S 687, S 690, S 697, S 702, S 703, S 706, S 738, S 745, S 801, S 878, S 884, S 892, S 898, S 905, S 916, S 922, S 956, S 961, S 971, S 974, S 977, S 994, S 1003, S 1008, S 1385, S 1394, S 1405, S 1421 et S 1464) et 18 un signum (S 594, S 677, S 684, S 717, S 795, S 864, S 890, S 939, S 950, S 1021, S 1031, S 1215, S 1220, S 1379, S 1454, S 1460, S 1471 et S 1473). À ce titre, seules quatre invocations ne se placent pas sous le nomen divin pour justifier l'action du roi : S 563, S 618, S 1225, S 1447.

204. 199 occurrences (27\%). Dans les chartes authentiques, on en compte 105 occurrences.

205. Sur 2800 monnaies dont l'avers est lisible, on en compte 109 qui utilisent l'expression totius Britanniae (un peu moins de $4 \%$ ). Néanmoins, presque toutes ont été frappées pendant le règne d'Æthelstan (95, soit un tiers de la frappe préservée de ce souverain), les autres appartenant aux monnayages d'Eadwig et Edgar.

206. S. D. Keynes, «Edgar... », p. 23-25 ; F. Barlow, Edward..., p. 135-137.

207. 12 occurrences $(2 \%)$. Dans les chartes authentiques, on en compte 8 occurrences : S 429, S 430, S 431, S 438, S 446, S 448, S 858 et S 877. 
dirigeant de populations qui vivent dans les zones voisines, in circuitu. C'est le cas, en particulier, dans le prologue de la Regularis Concordia, pour désigner le roi Edgar : "Christi opitulante gratia Anglorum ceterarumque gentium intra ambitum Britannicae insulae degentium rex egregius ${ }^{208}$.» Les peuples concernés sont désignés invariablement par les mots gens, populus, natio, plebs, voire intégrés à une regio ou une prouincia. Ce ressort vague est décrit par diverses formes adverbiales ou prépositionnelles : in circuitu, in gyro, circum, triviatim, undique, hic, hinc, etc. On en trouve des traces dans les chartes d'Offa de Mercie, dans les années $780^{209}$, ou d'Alfred ${ }^{210}$, mais dans des documents douteux. Bien qu'on les trouve dans quelques chartes authentiques d'Æthelstan ${ }^{211}$, leur usage massif appartient aux règnes d'Edmund et Eadred ( $72 \%$ et $67 \%$ ), à un moment où l'unité anglaise n'était pas assurée et où la menace iro-norvégienne en particulier était persistante ${ }^{212}$.

En tout et pour tout, 174 occurrences de ces formules peuvent être identifiées dans les chartes $(24 \%)^{213}$. Si cet usage se maintient après 955 , à hauteur de $20 \%$ environ des documents authentiques, le sens n'est sans doute pas le même : il s'agit plutôt d'affirmer une aire d'influence, au-delà des bornes du royaume des Anglais. Auquel cas, cette formule permettrait de maintenir une distinction commode entre le peuple auquel les rois appartiennent, celui sur lequel ils règnent, et les royaumes et peuples sur lesquels ils revendiquent une forme plus ou moins directe de domination, mais sans les reconnaître comme leurs ${ }^{214}$. Peut-être cette influence n'étaitelle que religieuse, comme l'indique l'usage de la formule in circuitu dans le prologue de la Regularis Concordia, auquel cas cette formule servirait à affirmer la primauté de l'archevêque de Canterbury sur les autres évêques insulaires et à appuyer le ministerium religieux du $\operatorname{roi}^{215}$. A contrario, la volonté d'estomper les frontières du royaume pour intégrer avec souplesse, de manière différenciée, les populations qui se trouvent au-delà de limites conventionnelles renvoie aussi aux prétentions universelles contenues dans les discours d'inspiration impériale.

208. Regularis concordia, Prologus (éd. T. Symons, Londres, 1953).

209. S 116 , S 117 et S 127.

210. S 351 et $\mathrm{S} 353$.

211. S 441 en 938. Mais aussi dans des chartes réputées douteuses : S 414, S 415, S 424 et $\mathrm{S} 442$.

212. Simon Keynes parle de « retenue » dans ces chartes (S. D. Keynes, « Edgar rex admirabilis... », p. 6).

213. 132 chartes authentiques (29\%).

214. R. DaVIES, The First English Empire..., p. 197-199.

215. E. John, Orbis Britanniae..., p. 15-16 ; F. BARLow, The English Church, 1000-1066. A History of the Later Anglo-Saxon Church, Londres, 1979, p. 235. 
Tandis que le royaume de Wessex absorbait ses voisins, battait ses ennemis et développait une influence importante sur le continent et dans les îles, la titulature royale se transforma. La titulature simple du milieu du $\mathrm{IX}^{\mathrm{e}}$ siècle s'adapta pour refléter cette évolution : les rois des West-Saxons sont devenus rois des Anglo-Saxons, puis rois des Anglais, voire rois de toute la Bretagne, de toute Albion, sinon imperatores, basileis ou recteurs des païens, des Northumbriens, des Bretons, des Danois et de peuples qui vivent dans les alentours.

Dans un premier temps, le titre changea à la marge, pour justifier l'incorporation des Merciens, en devenant rex Angul Saxonum. Mais ce titre incorpora presque aussitôt et rapidement de manière systématique le concept religieux et politique de gens Anglorum. Ce faisant, les rois des West-Saxons s'affirmaient comme les chefs d'un nouveau peuple élu, placé sous les auspices de Bède le Vénérable et, à travers lui, de Grégoire le Grand. Indirectement, cela leur permettait aussi d'incorporer tous les autres royaumes anglo-saxons sous leur bannière. Cette évolution, débutée sous Alfred le Grand, s'est close à l'époque d'Æthelstan, avant de devenir un standard dans le second $\mathrm{x}^{\mathrm{e}}$ siècle.

Lorsque le royaume des Anglais commença à s'étendre et à menacer ses voisins celtiques, jusqu'à revendiquer une forme d'hégémonie insulaire, les titres et les postures impériaux se multiplièrent et se complexifièrent : basileus, rex totius Britanniae. La référence faite aux antiques termes d'Albio et Britannia permettait de légitimer cet expansionnisme, de rendre compte d'une supériorité militaire passagère et de mettre en évidence une hégémonie plus ou moins reconnue. Ce recours à la géographie antique et à des territoires symboliques permettait de prendre appui sur des réalités incontestables ou de construire de nouveaux regroupements, qui font figure de leviers pour les desseins politiques de la monarchie anglosaxonne. Même si ce pouvoir est fluctuant et souvent fort théorique (rector et gubernator), que l'espace sous domination est difficile à délimiter avec précision (in circuitu), il se trouve que plusieurs peuples, plusieurs royaumes sont sous la domination du roi des Anglais, dans un espace qui va grandissant. La formulation, encore vague, tente néanmoins d'éviter l'opposition frontale avec les princes bretons insulaires.

Ainsi, les rois assument souvent un discours de nature impériale, lequel repose principalement sur un titre signifiant qui fait référence aux grands empires continentaux passés ou contemporains (basileus, primicerius, imperator, rector), la domination de peuples/royaumes divers (parfois mentionnés nominalement, mais souvent réunis implicitement sous des étiquettes très floues), dans un territoire vaste (qui inclut a minima l'île de Bretagne à compter du deuxième quart du $\mathrm{x}^{\mathrm{e}}$ siècle), sur la sacralité de leur pouvoir et sur un discours expansionniste. En cela toutefois quelques rois se distinguent. La plupart des innovations en la matière sont dues à 
Æthelstan, ont été renforcées et généralisées par Edgar, se sont maintenues au début du règne d'Æthelred II, avant de décliner jusqu'à l'arrivée au pouvoir de Cnut. Sous tous ces points de vue, il semble difficile de douter du fait qu'il y eut, dans le discours, une posture impériale de la part des rois. Cette posture et ce discours impériaux se sont fortement nourris des textes de Bède le Vénérable, tout en intégrant les modèles romain, byzantin et carolingien. La convergence assez nette des documents diplomatiques, normatifs et numismatiques témoignent du caractère singulier de cette construction, mais aussi du développement d'une " agence royale » capable de diffuser ce message et d'une acceptation de ce message chez les aristocrates qui produisirent une partie de ces documents.

Arnaud Lestremau - Collège Pablo Picasso (Garges-lès-Gonesse)/LAMOP (UMR 8589 - Paris 1 Panthéon-Sorbonne/CNRS)

\section{Basileus Anglorum. La prétention impériale dans les titulatures royales à la fin de la période anglo-saxonne}

Les titulatures reflètent l'étendue du pouvoir d'un roi, mais renvoient aussi l'image qu'il entend donner de lui-même. Dans une période de mutation politique rapide dans le monde insulaire, il est donc logique que ces titulatures évoluent elles aussi, pour mettre en lumière les conquêtes ou les annexions, tout en signalant les revendications territoriales. Au début $\mathrm{du} I \mathrm{X}^{\mathrm{e}}$ siècle, I'hégémonie mercienne se traduisait par l'utilisation de titres impériaux; à compter de la fin du siècle, les victoires décisives remportées par le Wessex permettent à ses rois d'assumer une titulature de plus en plus audacieuse. Rendant d'abord compte de l'incorporation dans un même royaume d'Angles et de Saxons, le roi Alfred se présente comme roi d'un nouveau royaume, celui des "Anglo-Saxons ". Reprenant ensuite l'étiquette ethnique unificatrice et salvatrice créée par Bède, lui et ses successeurs se présentent progressivement comme les rois des Anglais. Cette capacité à dominer plusieurs royaumes que tout séparait quelques décennies plus tôt, l'efficacité de la lutte contre les Vikings, les conquêtes réalisées et l'influence croissante du roi en dehors de son royaume lui permettent progressivement de passer une nouvelle étape, en adoptant des titres plus clinquants. Si l'on en croit la communication politique du Wessex aux $\mathrm{X}^{\mathrm{e}}-\mathrm{XI}^{\mathrm{e}}$ siècles, le royaume est devenu un empire, a minima pendant le règne des souverains les plus puissants, comme Æthelstan, Edgar ou Cnut.

Angleterre anglo-saxonne, chartes, communication politique, prétentions impériales, titulature

\section{Basileus Anglorum. Imperial claims in regnal styles at the end of the Anglo-Saxon period}

Regnal styles reflect the extent of a king's power, but they also show the image that he wants to project. During a period of intense political change in the British Isles, it was only logical that the regnal styles would change too, in order to highlight conquests and annexations and assert territorial claims. At the beginning of the ninth century, the Mercian hegemony was manifested by the use of imperial titles, and at the end of that century, the crucial victories of Wessex allowed their kings to assume increasingly audacious styles. As he had incorporated Angles into a Saxon kingdom, 
King Alfred presented himself as the king of a new kingdom, the kingdom of the Anglo-Saxons. He and his successors then took up a unifying and redeeming ethnic label forged by the Venerable Bede, and they progressively became the Kings of the English. Because they were able to rule several kingdoms that had been divided only a few decades earlier, and because of their ability to successfully fight against the Vikings, achieve conquests, and have a growing influence outside the kingdom, the kings chose even more daring and showy regnal styles. If one believes the political communication of Wessex during the tenth and eleventh centuries, the kingdom had become an empire, at the very least during the reign of its most powerful rulers, such as Athelstan, Edgar, or Cnut.

charters, imperial claims, late Anglo-Saxon England, political communication, regnal style 
